\title{
Laplacian-Level Quantum Hydrodynamic Theory for Plasmonics
}

\author{
Henrikh M. Baghramyan $\odot,{ }^{1}$ Fabio Della Sala๑ ${ }^{1,2}$ and Cristian Ciracì ${ }^{1, *}$ \\ ${ }^{1}$ Center for Biomolecular Nanotechnologies, Istituto Italiano di Tecnologia, \\ Via Barsanti 14, 73010 Arnesano (LE), Italy \\ ${ }^{2}$ Institute for Microelectronics and Microsystems (CNR-IMM), Via Monteroni, \\ Campus Unisalento, 73100 Lecce, Italy
}

(Received 5 June 2020; revised 21 October 2020; accepted 24 December 2020; published 11 March 2021)

\begin{abstract}
An accurate description of the optical response of subwavelength metallic particles and nanogap structures is a key problem of plasmonics. Quantum hydrodynamic theory $(\mathrm{QHT})$ has emerged as a powerful method to calculate the optical response of metallic nanoparticles (NPs) since it takes into account nonlocality and spillout effects. Nevertheless, the absorption spectra of metallic NPs obtained with conventional QHT, i.e., incorporating Thomas-Fermi (TF) and von Weizsäcker (vW) kinetic energy (KE) contributions, can be affected by several spurious resonances at energies higher than the main localized surface plasmon (LSP). These peaks are not present in reference time-dependent density-functional-theory spectra, where, instead, only a broad shoulder exists. Moreover, we show here that these peaks incorrectly reduce the LSP peak intensity and have a strong dependence on the simulation domain size so that a proper calculation of QHT absorption spectra can be problematic. In this article, we introduce a more general QHT method accounting for KE contributions depending on the Laplacian of the electronic density $(q)$, thus, beyond the gradient-only dependence of the TFvW functional. We show that employing a KE functional with a term proportional to $q^{2}$ results in an absorption spectrum free of spurious peaks, with LSP resonance of correct intensity and numerically stable Bennett state. Finally, we present a novel Laplacian-level KE functional that is very accurate for the description of the optical properties of NPs of different sizes as well as for dimers. Thus, the Laplacian-level QHT represents a novel, efficient, and accurate platform to study plasmonic systems.
\end{abstract}

DOI: 10.1103/PhysRevX.11.011049

Subject Areas: Computational Physics,

Condensed Matter Physics, Plasmonics

\section{INTRODUCTION}

Metal nanoparticles (NPs) play a crucial role in the enhancement of the optical field due to plasmonic effects [1], which make them an ideal platform for nonlinear optics $[2,3]$, hot-electron enhancement for photovoltaics [4,5], surface-enhanced Raman scattering [6], and imaging [7]. When it comes to the nanoscale, nonlocal and quantum effects play a crucial role in light-matter interaction [8]. Among theoretical approaches [9-17], time-dependent density-functional theory (TD-DFT) $[18,19]$ stands out since it allows us to accurately resolve the optical response of plasmonic structures at the nanoscale, including both quantum and atomistic effects [11,16,20-24]. However, TD-DFT is computationally expensive since all occupied orbitals need to be evaluated.

\section{*cristian.ciraci@iit.it}

Published by the American Physical Society under the terms of the Creative Commons Attribution 4.0 International license. Further distribution of this work must maintain attribution to the author(s) and the published article's title, journal citation, and DOI.
Another approach would be to treat the electron system semiclassically: a fluid characterized by the macroscopic local quantities, such as the electron density $n(\mathbf{r}, t)$ and the electron velocity field $\mathbf{v}(\mathbf{r}, t)$ [25-28], but at the same time considering quantum effects through energy functionals of the electron-density fluctuations. This approach is known as hydrodynamic theory (HT). The HT is part of a larger class of methods based on the orbital-free (OF) [29-31] description of quantum electronic systems dating back to the works of Thomas [32] and Fermi [33]. Although the interest in OF-DFT methods has gradually decreased in favor of Kohn-Sham (KS) orbital-based methods, the last decades have witnessed a reinvigorated interest due to the ideal scaling of computational resources with respect to the size of the electronic system offered by the OF-DFT approach [34]. Most of the research efforts in this field, however, have been devoted to static properties [35-38] and, more recently, also to response properties with the time-dependent OF-DFT [39-42]. In both cases, the central quantity that controls the accuracy of these methods is the noninteracting kinetic energy (KE) functional.

The most simple KE functional is the Thomas-Fermi (TF) functional, which accounts for the Pauli exclusion principle for a homogeneous system of noninteracting 
electrons [29], and it yields the electron quantum pressure $p(\mathbf{r}, t) \propto n(\mathbf{r}, t)^{5 / 3}$ [43] that accounts for the nonlocal electron response. It has been demonstrated that TF-HT is able to provide surprisingly accurate predictions that well match experiments with noble metal NPs, such as Au [44] and $\mathrm{Ag}$ [45], both qualitatively and quantitatively. Nevertheless, for alkali metals or aluminum, the TF-HT predicts a blueshift of the localized surface-plasmon resonance with respect to the classical Mie resonance [46], in contradiction with the redshift from the experiments [47] and TD-DFT calculations [48]. The origin of this difference lies in neglecting the spill out of the plasmon-induced charges at the NP surface [46]. In fact, the TF-HT approach employs (with some recent exception [49]) a spatially uniform electronic density inside the NP and zero outside (i.e., hard-wall boundary) [15].

To properly address spill-out effects, the spatial dependence of electron density as well as a correction to the KE functional, in order to describe the density variation effects, must be introduced. The simplest functional that depends on the gradient of the density is the von Weizsäcker (vW) functional $[29,50]$. The TF-HT with a fraction $(\lambda$, with $0<\lambda \leq 1$ ) of the $\mathrm{vW}$ correction (i.e., the $\mathrm{TF} \lambda \mathrm{vW} \mathrm{KE}$ functional) is usually referred to in the literature as the quantum hydrodynamic theory $(\mathrm{QHT})$ since the $\mathrm{vW}$ functional does not have a classical counterpart. The QHT has been largely used in plasma physics [51-56], magnetoplasmonics [57-59], plasmonic response properties of metal NPs of different geometries [60-66], as well as for surfaces [67-69] and strongly coupled plasmonic structures [65,70,71]. It has been shown that the QHT can predict plasmon resonance, spill out, and retardation effects in noble and simple metal NPs, matching very well with TD-DFT calculations $[62,65]$. There are also other works on the development of the QHT that consider the viscous contribution of electron fluid [70,72] and formulation of HT for nonlinear phenomena [73-75]. However, it is important to highlight that the QHT results depend on the approximation made for the KE functional (e.g., $\lambda$ parameter) as well as on the electronic density, which is an input quantity. The input electronic density can be obtained from a preceding OF-DFT calculation using the same KE functional used for the response, i.e., the self-consistent QHT (SC-QHT) approach of Ref. [61]. Other approaches use the exact KS density [62] or, more efficiently, a model density $[60,62]$ that reproduces the decay of the exact KS density.

Although QHT can describe different quantum effects relevant in plasmonics, it is not unaffected by drawbacks:

(i) Various TF-HT [76-78] and QHT [59,61-64,69] investigations for NPs [61,62], rods [63,64], and surfaces or slabs $[59,69,76-78]$ show the presence of one (or even more) additional resonances above the main plasmon peak and below the plasma frequency $\left(\omega_{p}\right)$. These resonances originate from the spatial variation of the electronic density as first pointed out by Bennett [76]. No Bennett states are observed when using hard-wall boundary conditions, both in the QHT [79-81] and in the TF-HT [81-84]: In these approaches, several peaks (volume plasmons) occur due to nonlocality but only at frequencies larger than $\omega_{p}$. Such Bennett states are thus peculiar to models with nonuniform density. In TD-DFT, a single Bennett state has been computed for jellium surfaces [85-87] at about $0.8 \omega_{p}$ in the case of sodium. Instead, for large jellium spheres, only a shoulder above the main plasmon peak is present [16] due to the interaction between single-particle transitions and surface modes [88-92].

To better illustrate this point, we anticipate in Fig. 1(a) the absorption spectra of one of the systems investigated in this work, i.e., a sodium jellium nanosphere. All calculations in this work focus on sodium (Wigner-Seitz radius $r_{s}=4$ a.u., with plasma frequency $\omega_{p}=5.89 \mathrm{eV}$ ), which is commonly investigated as a model metallic system. Figure 1(a) reports a direct comparison of the QHT with the TFvW functional (KS/TFvW denotes using $\lambda=1$ and the KS density as the input density) and reference TDDFT. TD-DFT can be considered as a reference for QHT because the latter method can be directly derived from TD-DFT equations (and for the two-electron case, the methods coincide) $[56,70,93]$. Although the energy position of the main peak, the localized surface plasmon (LSP), is very well reproduced, additional peaks are present in the QHT spectrum, which is not the case for the reference TD-DFT spectrum. The results in Fig. 1(a) represent the current state of the art of QHT calculations: Clearly, the presence of the other peaks strongly limits the QHT accuracy and applicability. Note that also the number and the position of the Bennett states strongly depend on the input density as well as on the $\lambda$ parameter $[69,77,78]$.

(ii) The QHT absorption spectrum of the metal nanoparticle is characterized by the critical frequency $[59,62,68]$

$$
\hbar \omega_{c}=\frac{\hbar^{2}}{m_{e}} \frac{\kappa^{2}}{8} \sqrt{\lambda}=|\mu| \frac{\sqrt{\lambda}}{\lambda_{g}},
$$

where $\kappa$ is the exponential decay constant of the ground-state density, $\lambda_{g}$ is the $\mathrm{vW}$ fraction used for the ground-state-density calculation $\left(\lambda_{g}=1\right.$ for exact KS density), $\lambda$ is the $\mathrm{vW}$ fraction used in the QHT response calculation, and $\mu=\lambda_{g}\left(\hbar^{2} \kappa^{2}\right) /\left(8 m_{e}\right)$ is the chemical potential [62]. For the KS calculations of the large $\mathrm{Na}$ jellium nanosphere, we have $\kappa \approx 1.05$ a.u. and $\mu \approx 3.75 \mathrm{eV}$ [62]. For energies above $\hbar \omega_{c}$, the induced density (i.e., the first-order change of the electronic density due to the excitation) has both an oscillating and exponentially decaying behavior, as 

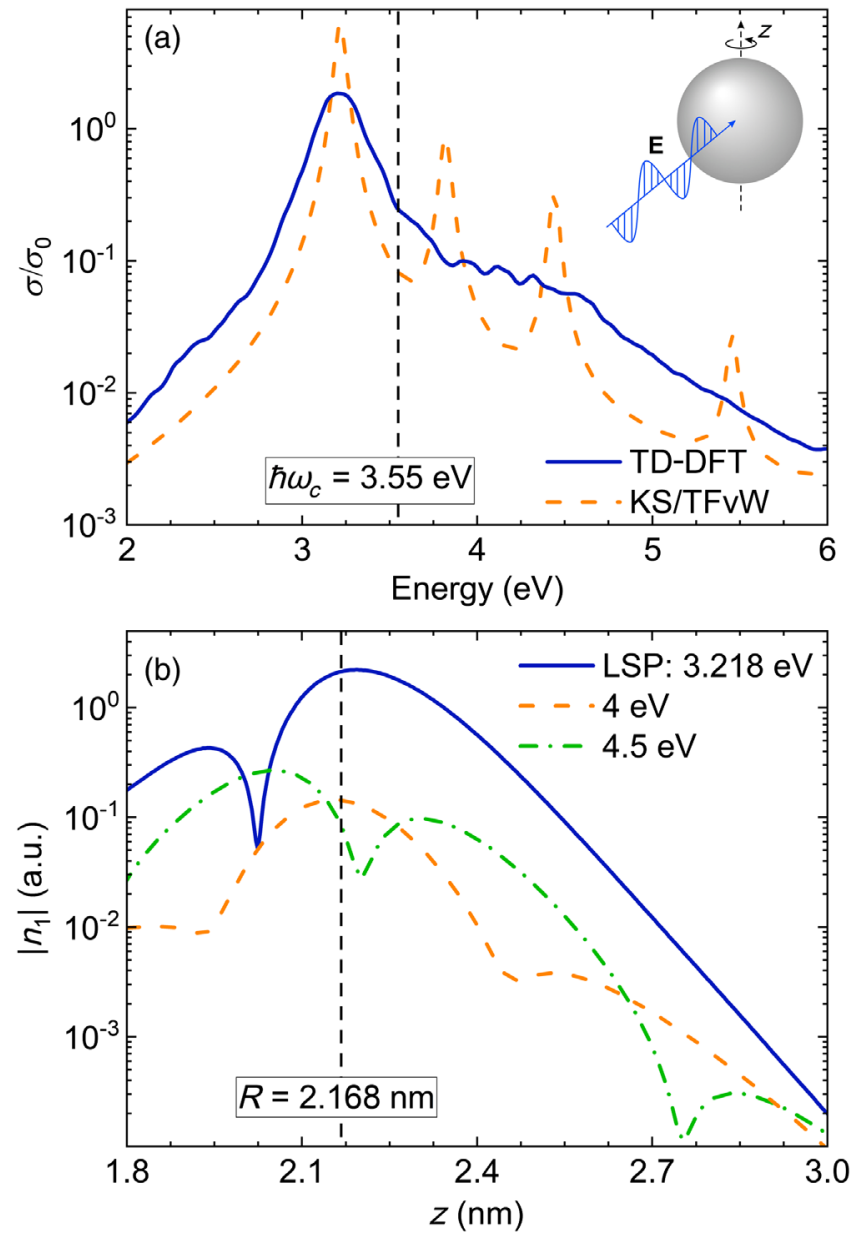

FIG. 1. (a) Normalized absorption cross section (in logarithmic scale) for a $\mathrm{Na}$ jellium sphere with radius $R=2.168 \mathrm{~nm}$ (and $N_{e}=1074$ electrons) as obtained from TD-DFT and QHT (using KS density and TFvW functional). $\hbar \omega_{c}$ is the critical frequency; see Eq. (1). (b) Induced charge density $n_{1}$ in atomic units (a.u.) at different energy excitations as calculated from QHT with the KS density and the TFvW functional. The inset in (a) schematically illustrates the nanosphere interaction with the incident plane wave. See the Appendix A for definitions and details on the absorption spectra calculation.

shown in Fig. 1(b), which is problematic to treat numerically. We show in this article that the energy position of all the peaks above the critical frequency strongly changes with the computational domain size so that a numerically converging QHT spectrum is challenging to obtain. The critical frequency can be artificially increased using an input density that decays faster (i.e., $\kappa>1.05$ or $\lambda_{g}<1$ ). In Ref. [61], for example, the SC-QHT approach with $\lambda_{g}=\lambda=$ $1 / 9$ (and thus, $\hbar \omega_{c}=3|\mu|$ ) was employed (i.e., the second-order gradient expansion [94]), leading to an input density decaying three times faster than the KS density, and thus strongly underestimating the spillout effects [62]. In the present work, we focus only on the more physical case of correct input density. (iii) The $\mathrm{TF} \lambda \mathrm{vW}$ functional is known to be quite a rough approximation of the exact KE, and different limitations of this functional have been shown in different contexts, e.g., lack of dynamical corrections $[40,55,68,95]$ and incorrect response for homogeneous electron gas $[30,96,97]$. Thus, the great accuracy of QHT calculations with the TF $\lambda \mathrm{vW}$ functional obtained in some cases [61,62] should be related to some error cancellation and, therefore, cannot have general validity.

In order to overcome these limitations, in this article, we extend the QHT approach to Laplacian-level KE functionals [94,97-105]. Laplacian-level KE functionals have been investigated in the past for ground-state properties, but with limited success [99-102]. Only recently, Laplacianlevel functionals performing well for semiconductors and metals in the framework of OF-DFT have been introduced [97,105]: The Pauli-Gaussian second-order and Laplacian (PGSL) functional has an improved Lindhard response [97], which is an important property for the description of metallic systems. Laplacian-level KE functionals are much simpler than fully nonlocal functionals based on the Lindhard response in the reciprocal space $[30,40,95,96]$ and can be easily applied to finite systems [97]. While Laplacian-level functionals have been applied for the ground-state properties, their application for optical properties is completely unexplored. In this work, we introduce the Laplacian-level QHT linear-response equations in the frequency domain. We carry out a general form of the QHT equations that holds for any arbitrary Laplacian-level functional, boosting the QHT potential in an unprecedented manner. We perform calculations for $\mathrm{Na}$ jellium nanospheres (up to 6000 electrons) and demonstrate that in the QHT-PGSL approach, only the main LSP peak appears in the lower part of the absorption spectrum, which is stable to the changes of computational domain size as well as on the input density. In fact, in QHT-PGSL, the induced density decays in the same way for all frequencies, and no critical frequencies exist anymore.

Finally, we go beyond the PGSL approximation and introduce the plasmonic tailored PGSLN functional, which gives very accurate plasmon energy, peak intensity, and Feibelman $d$-parameter [106], as well as a single numerically stable Bennett state. We present a detailed comparison of the different KE functional for QHT, and we clearly demonstrate that the QHT-PGSLN approach is the most accurate and numerically stable method to treat plasmonics nanosystems.

The article is organized as follows: In Sec. II, we introduce the equations governing the Laplacian-level QHT, which also contains the conventional QHT-TFvW approach as a special case. In Sec. III, we discuss theoretically the properties of the induced density in the tail region in spherical systems, showing that the QHT-PGSL has an unexpected 
and completely different behavior with respect to the conventional QHT. In Sec. IV, we provide numerical details of our implementation which can efficiently describe systems with spherical and cylindrical symmetry. In Sec. V, we compare in detail the absorption spectra of $\mathrm{Na}$ jellium nanospheres from TD-DFT, QHT-TFvW, QHT-PGS, and QHT-PGSL, showing their different dependence on the computational domain size as well as their oscillator strength. In Sec. VI, we describe the numerical results of the induced density decay for $\mathrm{Na}$ jellium nanospheres, which confirms the theoretical prediction of Sec. III. In Sec. VII, we present the derivation and the results for the PGSLN functional, which can be tuned to have a Bennett state at the correct energy. In Sec. VIII, we benchmark the energy, the oscillator strength, and the Feibelman $d$-parameter as a function of the particle size. In Sec. IX, we present the results for spherical dimers. Finally, the conclusion and future perspectives are drawn in Sec. X.

\section{LAPLACIAN-LEVEL FUNCTIONALS IN QUANTUM HYDRODYNAMICS}

The linearized QHT response $[28,107]$ is governed by the following equations [61,62] for the electric field $\mathbf{E}$ and polarization vector $\mathbf{P}$ :

$$
\begin{gathered}
\nabla \times \nabla \times \mathbf{E}-\frac{\omega^{2}}{c^{2}} \mathbf{E}=\omega^{2} \mu_{0} \mathbf{P} \\
\frac{e n_{0}}{m_{e}} \nabla\left(\frac{\delta G[n]}{\delta n}\right)_{1}+\left(\omega^{2}+i \gamma \omega\right) \mathbf{P}=-\varepsilon_{0} \omega_{p}^{2} \mathbf{E},
\end{gathered}
$$

where $c$ is the speed of light, $\varepsilon_{0}$ and $\mu_{0}$ are the vacuum permittivity and permeability, $m_{e}$ and $e$ are the electron mass and charge (in absolute value), $\gamma$ is the phenomenological damping rate, and $\omega_{p}(\mathbf{r})=\sqrt{e^{2} n_{0}(\mathbf{r}) /\left(m_{e} \epsilon_{0}\right)}$ is the plasma frequency with $n_{0}(\mathbf{r})$ being the ground-state (equilibrium) electron density. $(\delta G[n] / \delta n)_{1}$ is the firstorder term for the potential associated with the energy functional $G[n]$ given by

$$
G[n]=T_{s}[n]+E_{\mathrm{XC}}^{\mathrm{LDA}}[n],
$$

where $E_{\mathrm{XC}}^{\mathrm{LDA}}[n]$ is the exchange-correlation (XC) energy functional in the local density approximation (LDA), while $T_{s}$ is the noninteracting $\mathrm{KE}$ functional.

In general, the exact energy functional can be written as

$$
T_{s}[n]=\int\left[\tau^{\mathrm{TF}}(n)+\tau^{\mathrm{vW}}(n, w)\right] d^{3} \mathbf{r}+C_{s}[n]+C_{d}[n, \omega],
$$

where

$$
\tau^{\mathrm{TF}}(n)=\left(E_{h} a_{0}^{2}\right) \frac{3}{10}\left(3 \pi^{2}\right)^{2 / 3} n^{5 / 3}
$$

is the TF kinetic energy density (a simple local function of the electronic density), and

$$
\tau^{\mathrm{vW}}(n, w)=\left(E_{h} a_{0}^{2}\right) \frac{w}{8 n}
$$

is the $\mathrm{vW}$ term which depends on both $n$ and on the squared gradient of the density $w=\nabla n \cdot \nabla n$. In Eqs. (5) and (6), $E_{h}=\hbar^{2} /\left(m_{e} a_{0}^{2}\right)$ is the Hartree energy, and $a_{0}$ is the Bohr radius. Finally, $C_{s}$ and $C_{d}$ represent the generic density functionals for static and dynamic corrections, respectively. Although some schemes have been proposed [40,70,95], the first-principles derivation of dynamic corrections presents fundamental challenges, especially for finite-size systems.

In this article, we consider only static corrections. In particular, at the Laplacian level of theory, the KE has the form

$$
T_{s}[n]=\int \tau(n, w, q) d^{3} \mathbf{r},
$$

where the Laplacian of the density is $q=\nabla^{2} n$, which is a new ingredient in addition to $w$. The function $\tau(n, w, q)$ can be approximated in several ways [94,97-101]. In the PGSL functional [97], the function $\tau(n, w, q)$ is approximated as the sum of the vW [50], Pauli-Gaussian (PG $\alpha)$, and Laplacian $(\mathrm{L} \beta)$ terms [97]

$$
\tau(n, w, q)=\tau^{\mathrm{vW}}(n, w)+\tau^{\mathrm{PG} \alpha}(n, w)+\tau^{\mathrm{L} \beta}(n, q),
$$

where

$$
\begin{gathered}
\tau^{\mathrm{PG} \alpha}(n, w)=\tau^{\mathrm{TF}}(n) e^{-\alpha C n^{-8 / 3} w}, \\
\tau^{\mathrm{L} \beta}(n, q)=\beta \tau^{\mathrm{TF}}(n) q_{r}^{2}=\beta D n^{-5 / 3} q^{2},
\end{gathered}
$$

with the coefficients being $C=\left(3 \pi^{2}\right)^{-2 / 3} / 4$ and $D=$ $3\left(3 \pi^{2}\right)^{-2 / 3} E_{h} a_{0}^{2} / 160$.

In Eq. (9b), we also introduce the (adimensional) reduced Laplacian $[97,101,108]$, i.e., $q_{r}=3 q /\left(40 \tau^{\mathrm{TF}}\right)$, which is largely used for the development of $\mathrm{KE}$ functionals.

It is useful to identify the following cases:

(i) $\alpha=0, \beta=0$. Equations (3)-(9) reduce to the models employed in previous works $[62,65,68]$; i.e., $T_{s}[n]$ is approximated as the sum of the TF and $\mathrm{vW}$ functionals, and we indicate this case as TFvW (i.e., TF $\lambda \mathrm{vW}$ with $\lambda=1$ ).

(ii) $\alpha \neq 0, \beta=0$. It corresponds to the case where the QHT is improved with the addition of the $\mathrm{PG} \alpha$ functional. We refer to this case as QHT-PG $\alpha$.

(iii) $\alpha \neq 0, \beta \neq 0$. This is the more complex case in which the Laplacian-level correction $\mathrm{L} \beta$ is included in the energy functional. This case is referred to as QHT-PG $\alpha \mathrm{L} \beta$. 
The parameters $\alpha$ and $\beta$ can be determined in a nonempirical way by imposing exact asymptotic solutions. In particular, we set $\alpha=40 / 27$ in order to satisfy secondorder gradient expansion [97,109] and use PGS for PG40/ 27. Moreover, we follow the results of Ref. [97] and fix $\beta=0.25$ such that the overall correction functional PGSL0.25 accurately reproduces the linear-response function of a noninteracting homogeneous electron gas at both small and large wave vectors [97]. For brevity, we use the acronym PGSL for PGSL0.25.

In order to calculate the potential, we take the functional derivative of $T_{s}[n]$ [108] and obtain

$$
\begin{aligned}
\frac{\delta T_{s}}{\delta n}= & \tau_{n}+w\left(\tau_{n n q}-2 \tau_{n w}\right)+\left(\tau_{q n}-2 \tau_{w}\right) q \\
& +2\left(\tau_{n q q}-\tau_{w q}\right)(\nabla n \cdot \nabla q) \\
& +2\left(\tau_{n w q}-\tau_{w w}\right)(\nabla n \cdot \nabla w) \\
& +2 \tau_{w q q}(\nabla w \cdot \nabla q)+\tau_{w w q}|\nabla w|^{2} \\
& +\tau_{w q} \nabla^{2} w+\tau_{q q} \nabla^{2} q+\tau_{q q q}|\nabla q|^{2},
\end{aligned}
$$

where the subscripts $i=n, w, q$ denote the corresponding partial derivatives. The detailed derivation of Eq. (10) is given in Sec. I of the Supplemental Material [110]; a similar derivation can be found in Ref. [100].

While the kinetic potential in Eq. (10) is the key quantity for self-consistent OF-DFT calculations, it is not used in the QHT linear response, where, instead, the second-order functional derivative (never investigated so far) is required. In particular, the first-order term of the potential $\left(\delta T_{s} / \delta n\right)_{1}$ is required, and it can be obtained using a perturbation approach where the perturbed density is taken as $n=n_{0}+n_{1}$, with $n_{1}=(1 / e) \nabla \cdot \mathbf{P}$ being the electron density perturbation. After some tedious algebra and neglecting higher-order terms, we obtain the following expression for the linear potential (see Secs. I and II of the Supplemental Material for the full derivation [110]):

$$
\left(\frac{\delta T_{s}}{\delta n}\right)_{1}=\left(\frac{\delta T_{s}^{\mathrm{I}}}{\delta n}\right)_{1}+\left(\frac{\delta T_{s}^{\mathrm{II}}}{\delta n}\right)_{1}+\left(\frac{\delta T_{s}^{\mathrm{III}}}{\delta n}\right)_{1}
$$

where

$$
\begin{aligned}
\left(\frac{\delta T_{s}^{\mathrm{I}}}{\delta n}\right)_{1}= & \tau_{n n}^{(0)} n_{1}, \\
\left(\frac{\delta T_{s}^{\mathrm{II}}}{\delta n}\right)_{1}= & -2 \tau_{n n w}^{(0)}\left|\nabla n_{0}\right|^{2} n_{1}-2 \tau_{n w}^{(0)}\left[n_{1} \nabla^{2} n_{0}+\nabla n_{0} \cdot \nabla n_{1}\right]-2 \tau_{w}^{(0)} \nabla^{2} n_{1} \\
& -2 \tau_{w w}^{(0)}\left[2\left(\nabla n_{0} \cdot \nabla n_{1}\right) \nabla^{2} n_{0}+\nabla\left(\left|\nabla n_{0}\right|^{2}\right) \cdot \nabla n_{1}+2 \nabla n_{0} \cdot \nabla\left(\nabla n_{0} \cdot \nabla n_{1}\right)\right] \\
& -4 \tau_{w w w}^{(0)}\left(\nabla n_{0} \cdot \nabla n_{1}\right)\left[\nabla n_{0} \cdot \nabla\left(\left|\nabla n_{0}\right|^{2}\right)\right] \\
& -2 \tau_{n w w}^{(0)}\left\{2\left(\nabla n_{0} \cdot \nabla n_{1}\right)\left|\nabla n_{0}\right|^{2}+\left[\nabla n_{0} \cdot \nabla\left(\left|\nabla n_{0}\right|^{2}\right)\right] n_{1}\right\} \\
\left(\frac{\delta T_{s}^{\mathrm{III}}}{\delta n}\right)_{1}= & \tau_{n n n q}^{(0)}\left|\nabla n_{0}\right|^{2} n_{1}+\tau_{n n q}^{(0)}\left[2 \nabla n_{0} \cdot \nabla n_{1}+n_{1} \nabla^{2} n_{0}\right]+2 \tau_{n q}^{(0)} \nabla^{2} n_{1} \\
& +\tau_{n n q q}^{(0)}\left\{\left|\nabla n_{0}\right|^{2} \nabla^{2} n_{1}+2 n_{1}\left[\nabla n_{0} \cdot \nabla\left(\nabla^{2} n_{0}\right)\right]\right\} \\
& +\tau_{n q q}^{(0)}\left[\nabla^{2} n_{0} \nabla^{2} n_{1}+2 \nabla n_{1} \cdot \nabla\left(\nabla^{2} n_{0}\right)+2 \nabla n_{0} \cdot \nabla\left(\nabla^{2} n_{1}\right)+\nabla^{2}\left(\nabla^{2} n_{0}\right) n_{1}\right] \\
& +\tau_{q q}^{(0)} \nabla^{2}\left(\nabla^{2} n_{1}\right) \\
& +\tau_{q q q}^{(0)}\left\{2\left[\nabla\left(\nabla^{2} n_{0}\right) \cdot \nabla\left(\nabla^{2} n_{1}\right)\right]+\nabla^{2}\left(\nabla^{2} n_{0}\right) \nabla^{2} n_{1}\right\} \\
& +\tau_{n q q q}^{(0)}\left\{2\left[\nabla n_{0} \cdot \nabla\left(\nabla^{2} n_{0}\right)\right] \nabla^{2} n_{1}+\left|\nabla\left(\nabla^{2} n_{0}\right)\right|^{2} n_{1}\right\} \\
& +\tau_{q q q q}^{(0)}\left|\nabla\left(\nabla^{2} n_{0}\right)\right|^{2} \nabla^{2} n_{1} \cdot \\
&
\end{aligned}
$$


The superscript (0) indicates that the function is evaluated at $n=n_{0}$. The terms are grouped so that $\left(\delta T_{s}^{\mathrm{I}} / \delta n\right)_{1}$ includes only derivatives of $\tau$ with respect to $n,\left(\delta T_{s}^{\mathrm{II}} / \delta n\right)_{1}$ includes derivatives of $\tau$ with respect to $w$, and finally, $\left(\delta T_{s}^{\mathrm{III}} / \delta n\right)_{1}$ includes derivatives of $\tau$ with respect to $q$. Equation (12) thus represents a novel and a quite general expression for the QHT first-order potential with increasing complexity.

The term $\left(\delta T_{s}^{\mathrm{I}} / \delta n\right)_{1}$ is the only one included in the TFHT model, which is a local model. In the case of the TFvW functional, only the terms (12a) and (12b) survive. With the PGS functional, all terms in $\left(\delta T_{s}^{\mathrm{II}} / \delta n\right)_{1}$ are included, whereas PGSL includes all terms but (12j)-(121), as thirdand fourth-order derivatives of $\tau$ with respect to $q$ are not present in Eq. (8). The terms in Eqs. (12j)-(12l) are present in the functional described in Sec. VII, where the Laplacian term does not have a simple quadratic dependence on $q$.

Despite its apparent complexity, Eq. (12) can be implemented in finite-element codes. Moreover, we note that Eq. (12) is not the most general expression for a Laplacianlevel KE functional: When $\tau$ includes terms with products of $w$ and $q$, additional terms are present, which will be investigated elsewhere. Such a product is present in the fourth-order gradient expansion [98].

Finally, we recall that the first-order term $\left(\delta E_{\mathrm{XC}}^{\mathrm{LDA}}[n] / \delta n\right)_{1}$ for the XC potential can be obtained via the Perdew-Zunger LDA parametrization [111], and a its full expression can be found in Ref. [62].

\section{ASYMPTOTIC ANALYSIS}

As discussed in Ref. [62], the tail of the ground-state density plays a fundamental role in the determination of the QHT solutions. In this section, we summarize and generalize the derivation in Ref. [62] to a Laplacian-level functional. We start by taking the divergence of Eq. (2b), and we use the quasistatic approximation (so that $\varepsilon_{0} \nabla \cdot \mathbf{E}=\nabla \cdot \mathbf{P}=e n_{1}$ ), obtaining

$$
\begin{aligned}
\nabla & \cdot \frac{e n_{0}}{m_{e}} \nabla\left(\frac{\delta G}{\delta n}\right)_{1} \\
& =-\omega^{2} e n_{1}-\frac{e^{2}}{m_{e}}\left(\frac{e}{\varepsilon_{0}} n_{0} n_{1}+\nabla n_{0} \cdot \mathbf{E}\right) .
\end{aligned}
$$

To obtain the asymptotic form of Eq. (13), we assume that [62]

$$
\begin{gathered}
n_{0}(\mathbf{r}) \rightarrow A_{0} \exp (-\kappa r), \\
n_{1}(\mathbf{r}) \rightarrow B_{0} \exp (-\nu \kappa r) \cos (\theta),
\end{gathered}
$$

where $\kappa>0$ is the decay constant of the ground-state density, and $\nu \kappa$ is the decay constant of the (dipole excited) induced density.

The right-hand side (rhs) of Eq. (13) is asymptotically vanishing, and it decays as

$$
-\omega^{2} e n_{1}+\frac{3 e^{2} \kappa d_{1}}{4 \pi \epsilon_{0} m_{e}} \frac{n_{0} \cos (\theta)}{r^{3}}
$$

where $d_{1}$ is the dipole moment of $n_{1}$ (see Ref. [62]).

For the left-hand side (lhs), we first note terms like $\tau^{\mathrm{TF}}$, $\tau^{\mathrm{PG} \alpha}$, and the XC term will vanish exponentially [62]. Thus, in the case of the PGSL functional, we need to consider only $\tau^{\mathrm{vW}}$ and the new term $\tau^{\mathrm{L} \beta}$. For spherical systems, we have that the lhs of Eq. (13) can be written as

$$
\nabla n_{0}(r) \nabla\left(\frac{\delta T_{s}^{\mathrm{L} \beta}}{\delta n}\right)_{1}=\sum_{n=0}^{6} F_{k}\left[r, n_{0}(r)\right] \frac{d^{k} n_{1}(r)}{d r^{k}}
$$

where $n_{1}(\mathbf{r})=n_{1}(r) \cos (\theta)$ and $F_{k}$ are functions reported in Sec. III of the Supplemental Material [110]. Note for the PGSL functional, Eq. (17) involves derivatives of $n_{1}$ up to the sixth order. After some algebra (see Sec. III of the Supplemental Material [110]), we obtain

$$
\nabla n_{0} \nabla\left(\frac{\delta T_{s}^{\mathrm{vW}}}{\delta n}\right)_{1} \rightarrow\left(-\frac{\nu^{4}}{4}+\frac{\nu^{3}}{2}-\frac{\nu^{2}}{4}\right) \kappa^{4} n_{1}
$$

$$
\nabla n_{0} \nabla\left(\frac{\delta T_{s}^{\mathrm{L} \beta}}{\delta n}\right)_{1} \rightarrow \beta \frac{\sqrt[3]{3}}{\pi^{4 / 3}}\left(\frac{243 \nu^{6}-1377 \nu^{5}+2025 \nu^{4}+765 \nu^{3}-3885 \nu^{2}+2865 \nu-650}{19440} \kappa^{6}\right) \frac{n_{1}}{n_{0}^{2 / 3}} .
$$

Equation (18) has already been derived in Ref. [62], whereas Eq. (19) is a key finding of the present work. We recall that the terms with $E_{\mathrm{XC}}^{\mathrm{LDA}}$ and $T_{s}^{\mathrm{PG} \alpha}$ decay exponentially faster than $n_{1}$, and that Eqs. (18) and (19) represent only the leading terms in the asymptotic region.
When $\beta=0$, the PGSL functional is asymptotically equivalent to the $\mathrm{vW}$ functional: When $\hbar \omega$ is higher than critical energy [see Eq. (1)], the asymptotic decay is complex valued and oscillating. Otherwise, the asymptotic decay is exponential, and $\nu$ depends on $\omega$. 
When $\beta>0$, we find, interestingly, that the $\mathrm{L} \beta$ term gives an exponentially increasing contribution due to the division by $n_{0}^{2 / 3}$, which dominates over the term in Eq. (18) as well as the term on the rhs. Thus, the asymptotic solution does not depend on $\omega$, as in the conventional QHT approach with the TFvW functional, but it is related to the solutions of the sixth-degree polynomial in $\nu$ in Eq. (19), which are

$$
\begin{array}{lll}
-1.320, & +0.543, & +2 / 3, \\
+1.123, & +5 / 3, & +2.987 .
\end{array}
$$

Only for those values of $\nu$, the lhs term vanishes asymptotically, as does the rhs. Some of these solutions are not possible or unstable, i.e., those with $\nu \leq 2 / 3$, as the term $n_{1} / n_{0}^{2 / 3}$ will not decay asymptotically. The other three values of $\nu$ give the right asymptotic solution, but a highorder analytical analysis or a full numerical solution is required to select the actual value of $\nu$. Interestingly, all these solutions have $\nu>1$, which is another difference with respect to the QHT approach with the TFvW functional [62], where $\nu<1$.

\section{NUMERICAL IMPLEMENTATION}

The system of Eqs. (2) with Eq. (3) and expressions (12) is solved for a plane-wave excitation using a commercial implementation of the finite-element method (FEM) [112].

In order to easily compute absorption spectra for spheres and sphere dimers, we implement our equations using the $2.5 \mathrm{D}$ technique, which significantly reduces the computational time for axisymmetric structures [62,113,114]. A detailed explanation of the FEM implementation can be found in Appendix B. In particular, we use Dirichlet boundary conditions without making any assumptions of the asymptotic decay. A completely independent implementation has also been carried out using a finite-difference method for spherical systems in the quasistatic approximation: The results obtained with the two methods are numerically the same, and details of the finite-difference implementation will be published elsewhere.

In order to solve the system of Eqs. (2), an expression for the ground-state density function $n_{0}(\mathbf{r})$ is required. Throughout the article, we consider the following two ground-state density functions: (i) the exact KS density $n_{0}^{\mathrm{KS}}(\mathbf{r})$ calculated using a DFT in-house code [62], and (ii) a model density defined as $[60,62]$

$$
n_{0}^{\operatorname{Mod}}(r)=\frac{1}{1+\exp \left[\kappa^{\operatorname{Mod}}(r-R)\right]}
$$

normalized with a condition $\int n_{0}^{\operatorname{Mod}} d V=N_{e}$, where $N_{e}$ is the number of electrons. For the $\kappa^{\text {Mod }}$ coefficient, the $\kappa^{\mathrm{Mod}}=1.05 / a_{0}$ value is fixed and fitted with asymptotic decay of the KS electronic density decay [62]. Figure 2

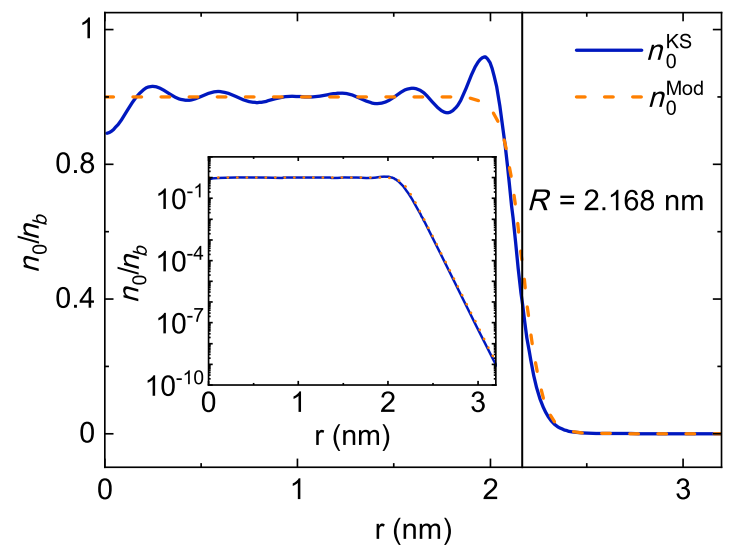

FIG. 2. $\mathrm{KS}\left(n_{0}^{\mathrm{KS}}\right)$ and model $\left(n_{0}^{\mathrm{Mod}}\right)$ ground-state densities for a Na jellium sphere with $N_{e}=1074$ electrons. The inset shows the variation of densities in the logarithmic scale. Values are normalized to the bulk density $n_{b}=\left(4 / 3 \pi r_{s}^{3}\right)^{-1}$.

shows $n_{0}^{\mathrm{KS}}$ and $n_{0}^{\mathrm{Mod}}$ densities for a $\mathrm{Na}$ (Wigner-Seitz radius $r_{s}=4$ a.u.) jellium nanosphere with $N_{e}=1074$ electrons (nanosphere radius $R=2.168 \mathrm{~nm}$ ). Note that $n_{0}^{\text {Mod }}$ does not display Friedel oscillations inside the nanosphere volume (surface marked with the vertical line), which are instead present in $n_{0}^{\mathrm{KS}}$. The inset shows that the asymptotic decay is the same for both cases.

\section{ABSORPTION SPECTRA}

In Fig. 3, we report the comparison of the normalized absorption cross section for a $\mathrm{Na}$ jellium nanosphere with $N_{e}=1074$ electrons as obtained using QHT with three different KE functionals (TFvW, PGS, PGSL) as well as the TD-DFT approaches (see Appendix A for definitions and

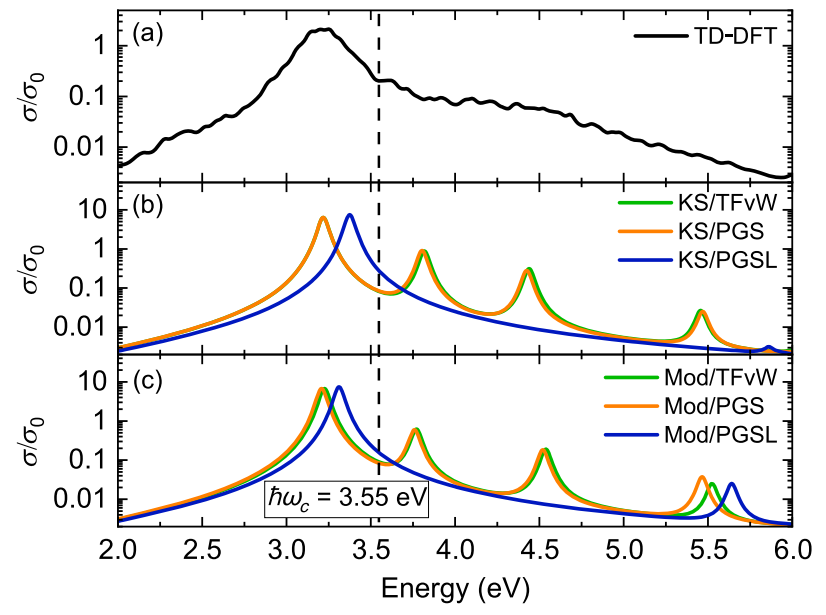

FIG. 3. Normalized absorption cross section $\sigma / \sigma_{0}$ (see the Appendix A for definitions) in logarithmic scale for a $\mathrm{Na}$ jellium sphere with $N_{e}=1074$ electrons as obtained from TD-DFT, QHT-TFvW, QHT-PGS, and QHT-PGSL using KS and model ground-state densities. $\hbar \omega_{c}$ is the critical frequency; see Eq. (1). 
details). The QHT results with a given KE functional $F$ are indicated in the following as $\mathrm{KS} / F$ or $\operatorname{Mod} / F$, if the $\mathrm{KS}$ density or the model density is used as the input density, respectively. When it is not relevant for the discussion to specify the input density, the shorthand QHT- $F$ is used.

Figure 3 shows that the energy of the LSP resonance (first main peak) for QHT-TFvW and QHT-PGS is in good agreement (within $10 \mathrm{meV}$ ) with TD-DFT (approximately $3.22 \mathrm{eV}$ ), which is broader due to quantum-size effects, while KS/PGSL and Mod/PGSL give the LSP peaks at approximately 3.37 and $3.31 \mathrm{eV}$, respectively, which are blueshifted with respect to TD-DFT results (for further analysis of the position of LSP peak, see Sec. VIII). As we discuss in the Introduction, QHT-TFvW gives accurate energy of the LSP and predicts additional peaks at higher energies, which are not present in the TD-DFT. Almost the same situation is obtained for QHT-PGS, meaning that even the more general gradient approximation in Eq. (9a) does not solve the problem of additional peaks. On the other hand, the QHT-PGSL absorption spectrum is quite different. The main difference between QHT-TFvW and QHTPGSL is not the energy shift of the LSP but the absence of additional resonances in the latter. Actually, a second small peak is present in the QHT-PGSL spectrum at high energy, namely, approximately $5.85 \mathrm{eV}$ [hardly visible in Fig. 3(b)] for KS/PGSL and approximately $5.7 \mathrm{eV}$ for Mod/PGSL. This peak is a Bennett state (which can be identified as shown in Fig. S6 of the Supplemental Material [110]), and it is further discussed in Sec. VII.

Although the QHT-TFvW predicts very well the LSP resonance when compared to more sophisticated TD-DFT approaches, the presence of additional peaks is a major shortcoming. These peaks are, in fact, very sensitive to the details in the tail of the density (see Fig. S4 in the Supplemental Material [110], where a model density with different $\kappa^{\text {Mod }}$ are considered). A small modification of the tail of the input density should not change the absorption spectrum significantly. This is the case for the Mod/PGSL absorption spectra, which are thus robust with respect to the input density. On the other hand, the Mod/TFvW absorption spectrum is instead very sensitive, and it results in being largely affected by additional peaks. These peaks have an energy higher than $\hbar \omega_{c}$ and can hardly be treated in an efficient numerical scheme.

This behavior is shown in Fig. 4, where the QHT-TFvW normalized absorption cross sections $\left(\sigma / \sigma_{0}\right)$ for the same jellium nanosphere are calculated for increasing size of the simulation domain. These calculations have been done with an in-house-developed finite-difference code for spherical systems (see Sec. IV), which reproduces exactly the FEM results reported in this work but is more accurate in the asymptotic region (since it requires only a one-dimensional discretization). The results are obtained with KS (upper panel) and model (lower panel) ground-state densities. Clearly, as the domain size increases, more and more

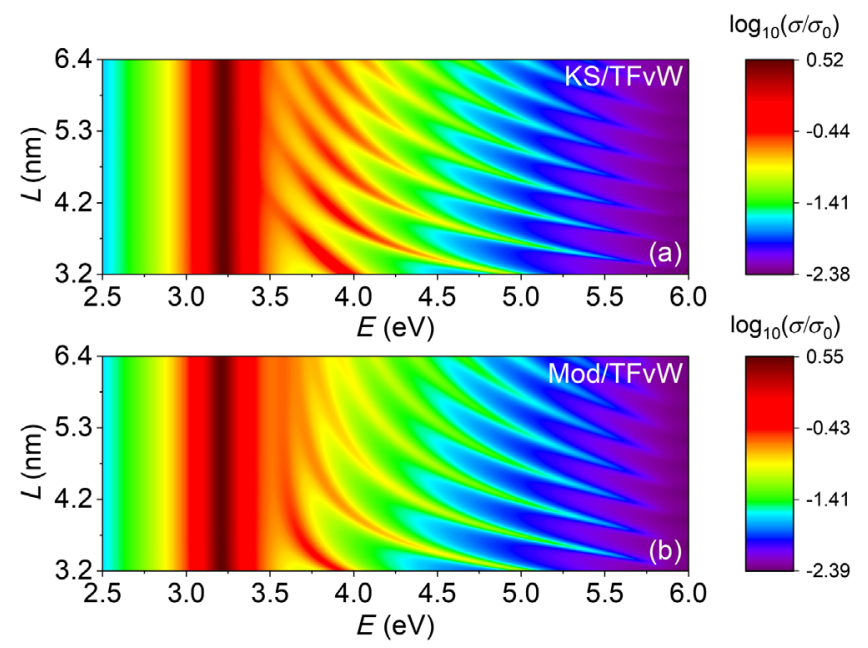

FIG. 4. The effect of computational domain size $(L)$ on the normalized absorption spectra as obtained from KS/QHT (a) and Mod/QHT (b) for a $\mathrm{Na}$ jellium nanosphere with $N_{e}=1074$ electrons.

modes appear (and with reduced intensities) in the spectrum without any limit. Thus, the absorption spectrum is very sensitive to the domain size. We note that no previous report in the literature has considered the numerical convergence of those states in QHT calculations. With an infinite computational domain size, there should be an infinite number of states with infinitely small peak intensity; i.e., no peaks can be distinguished anymore, and only an unstructured shoulder could be present.

This behavior is indeed shown in Fig. 5 where we report the QHT and TD-DFT results for two different computational domain sizes. We use a larger damping (see Eq. (2b) and

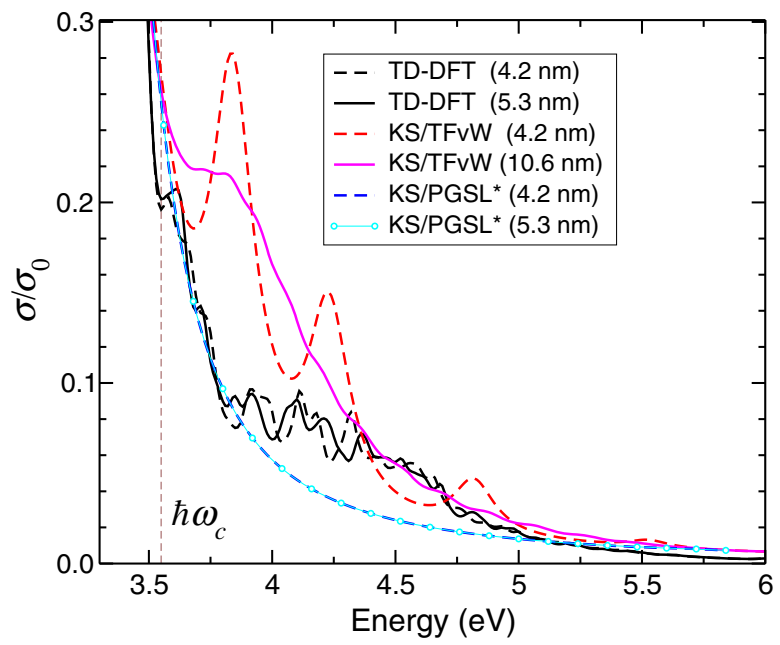

FIG. 5. High-energy part of the normalized absorption cross section for $N_{e}=1074$ electron $\mathrm{Na}$ jellium nanosphere considering different computational domain sizes for QHT with different functionals and TD-DFT. KS/PGSL* means that the spectrum has been redshifted in order to have the same energy position as KS/TFvW. The damping is $\gamma=0.2 \mathrm{eV}(\gamma=0.234 \mathrm{eV})$ for $\mathrm{KS} / \mathrm{TFvW}$ (KS/PGSL*). $\hbar \omega_{c}$ is the critical frequency; see Eq. (1). 


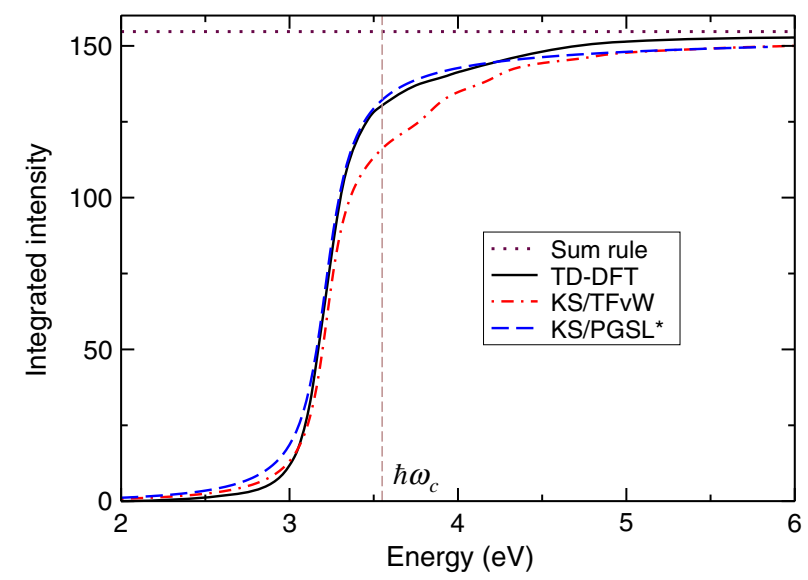

FIG. 6. Integrated intensity from KS/TFvW, KS/PGSL*, and TD-DFT for a Na jellium nanosphere with $N_{e}=1074$ electrons. The classical limit is also reported.

Appendix A) for KS/TFvW (namely, $\gamma=0.2 \mathrm{eV}$ ) so that it will give the same intensity at the LSP peak as TD-DFT. While the TD-DFT results are converged with standard domain size, convergence seems to appear for $\mathrm{KS} / \mathrm{TFvW}$ only with a domain size of $10.6 \mathrm{~nm}$ (200 a.u.), where no more Bennett peaks can be distinguished and only a shoulder is present. However, this shoulder, which starts at $\hbar \omega_{c}$, is significantly higher (about a factor of 2.5 in intensity) than the TD-DFT one, which starts later at about $3.7 \mathrm{eV}$. Clearly, a domain size of 200 a.u. to obtain a converged absorption spectrum is not reasonable for any application in plasmonics, and it is obtained only with a specialized code for reference calculations.

In Fig. 5, we also report the KS/PGSL* results, where the * indicates that the spectra are redshifted by $0.15 \mathrm{eV}$ in order to have the same LSP energy position as QHT; the damping is fixed to $\gamma=0.234 \mathrm{eV}$ so that the peak intensity is also the same. The plot shows that the KS/PGSL* does not change at all with the computational domain size (see also Fig S5 in the Supplemental Material [110]), and overall it is much closer to TD-DFT than QHT.

A more quantitative comparison of methods can be done by considering the integrated absorption cross section

$$
I(\omega)=\int_{0}^{\omega} \sigma\left(\omega^{\prime}\right) d \omega^{\prime}
$$

which converges to $\left(\pi e^{2}\right) /\left(2 \epsilon_{0} m_{e} c\right) N_{e}$ for $\omega \rightarrow \infty$, where $N_{e}$ is the number of electrons $[89,115,116]$.

The integrated absorption is plotted in Fig. 6, and it shows that $I(\omega)$ for QHT-TFvW and QHT-PGSL* converge to the same value for high energies. However, while the integrated absorption curve for TD-DFT and QHTPGSL* are very close to each other, the growth in QHT$\mathrm{TFvW}$ is much slower, meaning that the oscillator strength (i.e., the energy-integrated intensity) in QHT-TFvW is split into several Bennett modes, whereas the single peak in
QHT-PGSL* contains it all. In fact, the integrated absorption for QHT-TFvW at $\hbar \omega=\hbar \omega_{c}$ is about $15 \%$ smaller than QHT-PGSL* and TD-DFT.

In Sec. VIII, a more detailed analysis of the oscillator strength and absorption cross section for different numbers of electrons is presented. Here, we remark that these features are not limited to spherical NPs but could happen in other geometries or materials. In fact, for $\omega_{c}$ the identical expression was obtained for a jellium sphere [62] and slab [68]. Thus, in general, one could have for LSP $\omega_{\mathrm{LSP}} \simeq \omega_{c}$ or even $\omega_{\mathrm{LSP}}>\omega_{c}$. In such cases, the QHT cannot describe the LSP peak (see also Fig. S4 in the Supplemental Material [110]).

\section{INDUCED CHARGE DENSITY}

As we discuss in Sec. III, the decay of QHT-TFvW induced densities is frequency dependent, and solutions are pure exponentially decaying at the metal surface only if the incident plane-wave energy is lower than $\hbar \omega_{c}$, whereas using the PGSL functional, a fixed exponential decay should be obtained.

This fact can be verified numerically by plotting the computed induced charge density $n_{1}$ (associated with the absorption). In Fig. 7, we plot $\left|n_{1}\right|$ (in logarithmic scale) as obtained from the KS/TFvW and KS/PGSL for a Na jellium nanosphere with $N_{e}=1074$. To have a clear comparison of decay rates, the curves for $\left|n_{1}\right|$ are shifted to have the maximum at $z=R$ and normalized to $\left|n_{1}(R)\right|$, while $n_{0}$ density is normalized only to $n_{0}(R)$.

For the KS/TFvW induced density, the decay slope shows a clear dependence on the incident energy $\hbar \omega$, becoming oscillatory for $\hbar \omega>\hbar \omega_{c}=3.55 \mathrm{eV}$ (note that $\mathrm{KS} / \mathrm{TFvW}$ induced densities are not converged with respect to the computational domain size, as we discuss in Sec. V).

On the other hand, the KS/PGSL calculations yield the same slope for all excitation energies, as we analytically demonstrate in Sec. III. A numerical fit of the decay gives a value of $\nu$ close to +1.12 , i.e., the slowest from asymptotically decaying solutions (with $\nu>2 / 3$ ), see Eq. (20).

It is important to note that the TD-DFT calculations [Fig. 7(c)] give qualitatively similar results to the QHTPGSL. In fact, for TD-DFT we get the same decay slope for the induced density (at least for $\hbar \omega<\hbar \omega_{p}$ ). However, as we discuss in Sec. III, in QHT-PGSL we have $\nu>1$, while $\nu<1$ in the QHT-TFvW, meaning that spill-out effects are somehow smaller in QHT-PSGL.

Nonetheless, we need to point out that this feature is peculiar to PGSL, which is one of the few Laplacian-level KE functionals, and PGSL has not been developed for QHT calculations. Thus, another Laplacian-level KE functional can be developed with different features. In Sec. VIII, the induced charge density is further analyzed in terms of Feibelman $d$-parameters.

Another important aspect is the numerical stability of the QHT-PGSL approach: Not only the absorption spectra do 

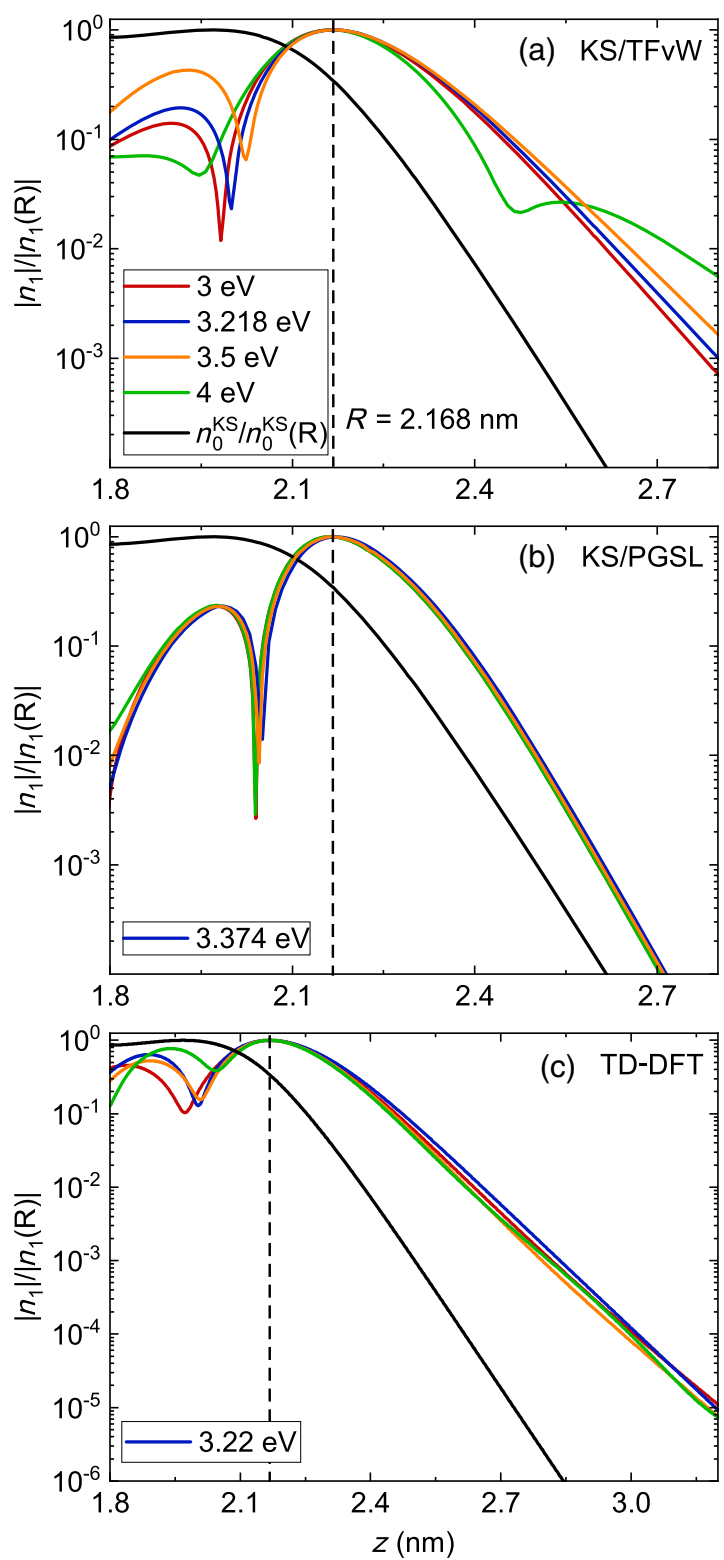

FIG. 7. Modulus of induced charged density at different energies for a Na jellium sphere with $N_{e}=1074$ electrons as calculated from KS/TFvW (a), KS/PGSL (b), and TD-DFT (c). The blue curves correspond to the densities associated with the LSP excitation energy. The critical frequency is $\hbar \omega_{c}=3.55 \mathrm{eV}$; see Ref. [62].

not depend on the domain size, but the fact that the decay constant is fixed and independent of the frequency allows the use of the mixed boundary condition for an exponential decay (i.e., $\hat{r} \cdot \nabla n_{1}+\nu \kappa n_{1}=0$ ), allowing converged results even with a very small domain size (see Fig. S5 in the Supplemental Material [110]).

\section{TOWARD AN ACCURATE KINETIC ENERGY FUNCTIONAL FOR QHT}

In the previous sections, we show that the QHT results with the PGSL functional are distinctively different from the ones obtained with the more conventional $\mathrm{TFvW}$ and PGS functionals. In particular, the PGSL functional modifies the description of the density tail, removes all the additional high-energy peaks, and improves the oscillator strength of the LSP peak, but it overestimates its energy. We recall that the PGSL functional has not been developed for QHT linear response but for ground-state OF-DFT calculations of bulk properties of metal and semiconductors [97]. Nevertheless, we show in the previous sections that the Laplacian term (i.e., $\beta q^{2}$ ) present in the PGSL functional is of fundamental importance also for QHT. In this section, we propose a modification of the PGSL functional to describe accurately the QHT linear-response properties. We find that a modification of the $\beta$ parameter does not lead to any relevant modification of the results. This can be understood considering that the asymptotic solutions [i.e., Eq. (20)] do not depend on $\beta$.

Here, we consider the following kinetic energy density (named PGSLN):

$\tau=\tau^{\mathrm{vW}}+\tau^{\mathrm{PGS}}+\tau^{\mathrm{TF}}\left[\beta q_{r}^{2}+2 \beta q_{0}^{2} \ln \left(1+q_{r} / q_{0}\right)\right]$,

where $q_{0}$ is a parameter. In this way, for large $q_{r}\left(q_{r} \gg q_{0}\right)$, i.e., in the density tail, the functional will be equivalent to PGSL, whereas for $q_{r} \ll q_{0}$ (i.e., inside the nanoparticle, where $\left|q_{r}\right|<0.2$; see Fig. S7 of the Supplemental Material [110]), we have that

$$
\tau \approx \tau^{\mathrm{vW}}+\tau^{\mathrm{PGS}}+2 q_{0} \beta q_{r}+O\left(q_{r}{ }^{3}\right),
$$

thus removing the quadratic term $q_{r}{ }^{2}$. For small $q_{r}$, the PGSLN functional will be thus equivalent to PGS because a linear term in $q_{r}$ does not contribute to the kinetic energy or to the kinetic potential [117]. The PGSLN is an accurate total kinetic energy functional yielding also accurate total energies of jellium nanospheres; see Table S2 of the Supplemental Material [110]. The parameter $q_{0}$ has a well-defined physical meaning, as it defines how rapidly the PGSL behavior is recovered at the density tail: The larger $q_{0}$, the further the quadratic term $q_{r}^{2}$ is recovered. The PGSLN is thus an interpolation between two density regimes where exact conditions are known; the asymptotic region where the quadratic term $q_{r}^{2}$ will render the induced density decay independent of the frequency (see Sec. III) and the region inside the nanoparticle where the density is slowly varying and the PGS functional satisfies the secondorder gradient expansion [97]. The transition between these two density regimes is described by the $q_{0}$ parameter, whose actual value is defined in the following.

In Fig. 8, we report the absorption spectrum as computed from TD-DFT, KS/PGSL, and KS/PGSLN using a larger damping for PGSL and PGSLN in order to have the same intensity for the LSP peak.

The first main difference between KS/PGSL and KS/PGSLN is the presence of a well-defined second 


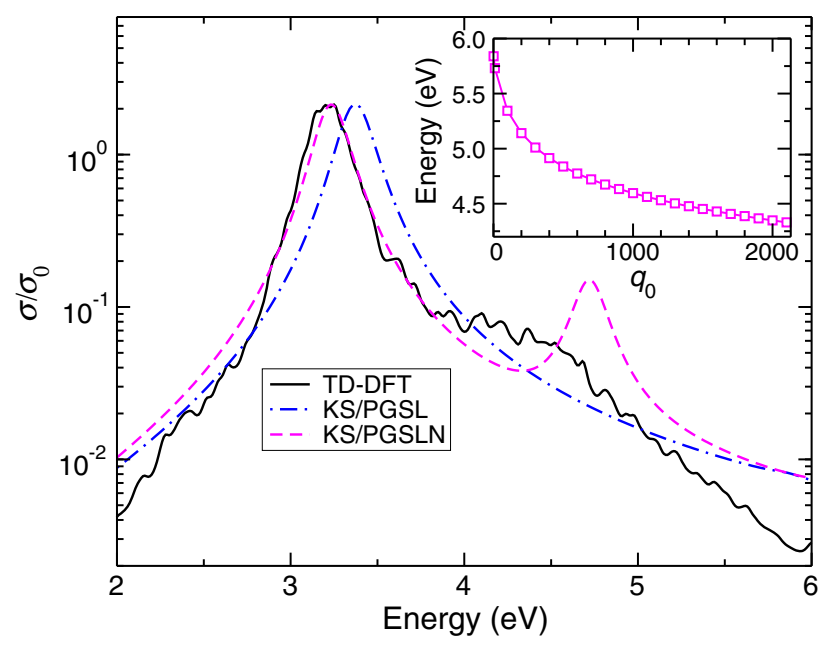

FIG. 8. Normalized absorption cross section (in logarithmic scale) for a $\mathrm{Na}$ jellium nanosphere with $N_{e}=1074$ electrons as computed from TD-DFT, KS/PGSL, and KS/PGSLN with $q_{0}=700$. The damping is $\gamma=0.234 \mathrm{eV}(\gamma=0.224 \mathrm{eV})$ for KS/PGSL (KS/PGSLN) so that all spectra have the same intensity at the LSP peak. In the inset is the position of the Bennett peak for different values of $q_{0}$ for the PGSLN functional.

(Bennett) peak at $4.7 \mathrm{eV}$ (see also Fig. S6 in the Supplemental Material [110]). For KS/PGSL the Bennett peak (at $5.85 \mathrm{eV}$ ) cannot be distinguished at all when a large damping is used. The KS/PGSLN spectra are stable with respect to the computational domain size (see Fig. S5 in the Supplemental Material [110]), but the energy position of the Bennett state changes with the values of $q_{0}$, as shown in the inset. When $q_{0} \approx 0$, the PGSLN functional is close to PGSL and indeed the position of the Bennett peak is at very high energy (close to the volume plasmons, as also shown in Fig. 3). Larger $q_{0}$ gives smaller energy of the Bennett peak. We define the PGSLN functional with $q_{0}=700$ a.u. in order to have the Bennett peak at $4.7 \mathrm{eV}$, as obtained from TD-DFT calculations for $\mathrm{Na}$ metal surfaces $[85,86]$. Fixing $q_{0}=700$ a.u. means that the PGSLN functional recovers the PGSL behavior only quite far outside the nanoparticle (see Fig. S7 of the Supplemental Material [110]). Note that fixing the parameters from the reference calculations of model systems is a standard procedure for DFT functional development since the known exact conditions are usually insufficient to build the full functional $[99,108,118]$.

As stated in the Introduction, in TD-DFT calculations of nanoparticles, a well-defined Bennett state is not present, because it is strongly damped and broadened due to the interaction with single-particle transitions (not included, by definition, in any hydrodynamical approach). Thus, the overall agreement between the PGSLN and TD-DFT spectra is not very good in the high-energy part (a sharp peak is present in QHT-PGSLN, whereas TD-DFT shows only a broad shoulder). A possible solution to be investigated in the future is to use in QHT a frequency-dependent

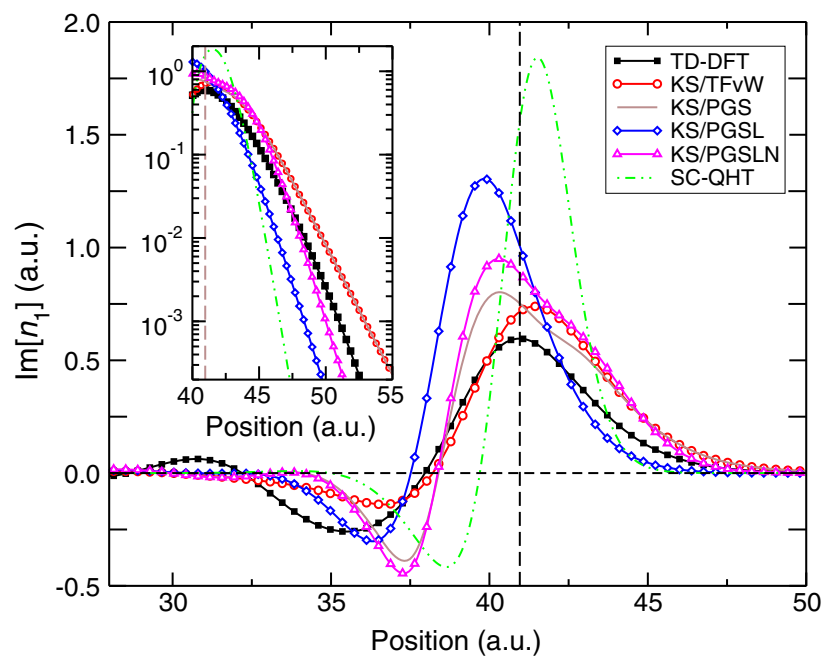

FIG. 9. Imaginary part of the induced density for a Na jellium nanosphere with $N_{e}=1074$ as computed for TD-DFT, KS/ TFvW, KS/PGS, KS/PGSL, KS/PGSLN, and SC-QHT. The inset shows the tail region in logarithmic scale.

damping (in contrast to a fixed value employed here) so that the QHT Bennett peak could be made broader (as it is in TD-DFT).

The second important difference is that the position of the LSP in PGSLN is redshifted to the correct (i.e., TDDFT) position. Thus, the PGSLN functional not only predicts a correct and numerically stable Bennett peak, but it also corrects the overestimation of the LSP energy, peculiar to the PGSL functional. Thus, the selected value of $q_{0}$ defined from the position of the Bennett state also yields a LSP energy in excellent agreement with TD-DFT results. This result can be seen as an independent check of the reliability of the $q_{0}$ parameter, and it is an important result as the $q_{0}$ parameter could be also defined to reproduce the LSP peak energy position: In this case, as a direct consequence, the Bennett state will be at the right energy. Thus, the $q_{0}$ parameter is not a bare empirical parameter, but it describes the interplay between the Bennett state, which is related to the density decay far away from the system, and the LSP peak, which is instead related to density behavior inside the nanoparticle. In Sec. VIII, a detailed benchmark on the LSP energy position for $\mathrm{Na}$ jellium spheres of different dimensions is presented.

Finally, in Fig. 9, we consider the induced density $n_{1}$ for different functionals. Figure 9 shows that KS/TFvW and KS/ PGS give a quite accurate description of the induced density as compared to TD-DFT, but with an asymptotic tail (see the inset), which is slower than TD-DFT. KS/PGSL is instead more confined inside the nanoparticles and decays faster (see also Fig. 7). The KS/PGSLN induced density is instead close to the KS/PGS one inside the nanoparticle, whereas in the tail, it approaches KS/PGSL. In Fig. 9, we also report the induced density from the SC-QHT approach: The shape of the $n_{1}$ is very different from all other QHT and TD-DFT 
results, as SC-QHT uses the self-consistent OF-DFT density as the input density, which is very different from the exact $\mathrm{KS}$ density. More quantitative analysis with the Feibelman $d$ parameter is given in Sec. VIII.

\section{BENCHMARKING KINETIC ENERGY FUNCTIONALS FOR JELLIUM NANOSPHERES OF DIFFERENT DIMENSIONS}

An important aspect in nanoplasmonic systems is the LSP resonance dependence on the NP size $[47,48,119]$. In Fig. 10(a) (horizontal axis is in logarithmic scale), we show the LSP resonance energy of various Na jellium nanospheres with the number of electrons $N_{e}$ varying from 338 to 6174 (the corresponding radius is $R=r_{s} N_{e}^{1 / 3}$ ) as computed from TD-DFT, KS/TFvW, KS/PGS, KS/PGSL, and KS/PGSLN. We see that for all approaches, LSP energy is lower than Mie theory value $\hbar \omega_{\text {Mie }}=3.4 \mathrm{eV}$ (shown as a horizontal line) and approaching it for large $N_{e}$. The mean average errors (MAEs) with respect to reference TD-DFT are reported in the first part of Table I for both KS and model density. Note that a detailed comparison of QHT method vs TD-DFT can only be achieved using the KS density. In fact, the model density is not the one used for the reference TD-DFT calculations. In any case, for applications involving large systems, the model density is simpler to use; thus, it is relevant to verify (even if approximately) its accuracy.

Figure 10(a) and Table I show that the accuracy of KS/ $\mathrm{TFvW}$ is very high $(\mathrm{MAE}=18 \mathrm{meV})$, which is somehow surprising, considering the shortcomings of the TFvW functional discussed in the Introduction. For Mod/TFvW, the accuracy is even higher (MAE $=6 \mathrm{meV}$, close to the numerical accuracy of our implementation). The PGS
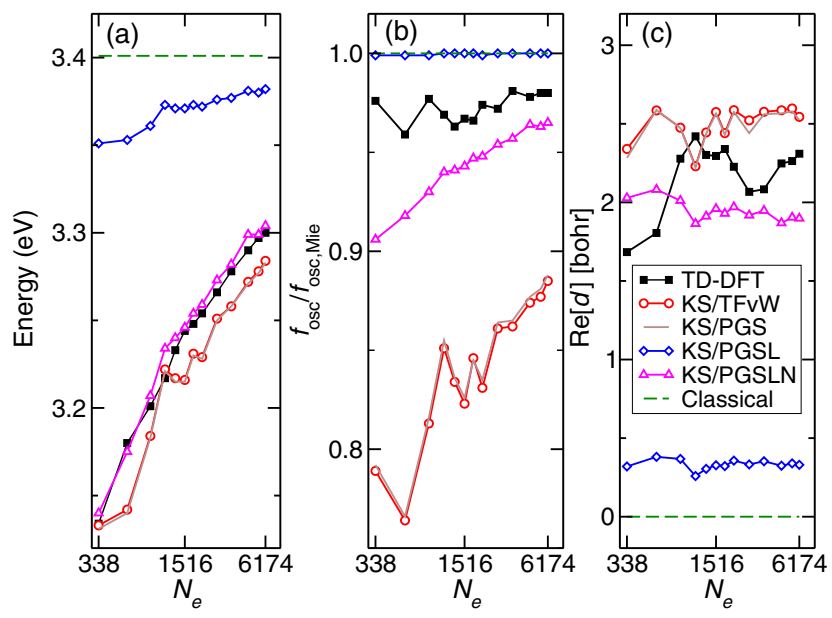

FIG. 10. (a) LSP energy, (b) corresponding oscillator strength $\left(f_{\text {osc }}\right)$ normalized to the Mie one, and (c) $d$-parameter (real part) at the LSP energy for $\mathrm{Na}$ jellium spheres as a function of the number of electrons $\left(N_{e}\right)$ as computed from TD-DFT, KS/TFvW, $\mathrm{KS} / \mathrm{PGS}, \mathrm{KS} / \mathrm{PGSL}$, and KS/PGSLN, as well as the classical results.
TABLE I. Performance of the QHT approach using different kinetic energy functionals (TFvW, PGS, PGSL, and PGSLN) with different input density (KS, model). The first block reports the mean absolute error (MAE) in meV for the energy position of the main LSP peak. The second block reports the mean absolute relative error (MARE) in percent for the oscillator strength of the main LSP peak. The last block reports the MAE (in a.u.) for the Feibelman $d$-parameter (real part) as computed from the induced density at the LSP energy. Best results (or close to them) are in bold; worst results (or close to them) are underlined.

\begin{tabular}{|c|c|c|c|c|}
\hline Density & TFvW & PGS & PGSL & PGSLN \\
\hline \multicolumn{5}{|c|}{ LSP, MAE (meV) } \\
\hline KS & 18 & 19 & 129 & 6 \\
\hline Mod & 6 & 12 & $\underline{80}$ & 14 \\
\hline \multicolumn{5}{|c|}{$f_{\text {osc }}, \operatorname{MARE}(\%)$} \\
\hline KS & 13.7 & 13.4 & 2.8 & 2.9 \\
\hline Mod & $\underline{6.3}$ & $\underline{6.1}$ & 2.4 & 0.8 \\
\hline \multicolumn{5}{|c|}{$\operatorname{Re}[d]$, MAE (bohr) } \\
\hline KS & 0.35 & 0.33 & 1.84 & 0.32 \\
\hline Mod & 0.19 & 0.15 & 1.26 & 0.41 \\
\hline
\end{tabular}

functional, which has some better properties than the TFvW functional [97], yields similar accuracy. On the other hand, PGSL overestimates the LSP peak by $80 \mathrm{meV}$ for the model density and $129 \mathrm{meV}$ for the KS density. This seems like quite a large error, but it is not if we consider that the widely used TF-HT has an error of $287 \mathrm{meV}$ on a similar test set [62]. The larger error of KS/PGSL with respect to Mod/PGSL can be traced back to the higher oscillating behavior of the KS density inside the NP (see Fig. 2). Such quantum oscillations induce higher values of the Laplacian (see Fig. S7 in the Supplemental Material [110]) and thus higher contributions to the energy and potential. On the other hand, with the model density, both the gradient and the Laplacian are vanishing small inside the NP. An "exact" KE functional should be able to describe both situations, but this is not the case of the PGSL functional, which has not been optimized for the jellium nanosphere or for the QHT approach. Instead, a properly constructed functional like PGSLN has even better accuracy than KS/TFvW: The MAE of KS/PGSLN is, in fact, only $6 \mathrm{meV}$.

To describe the accuracy of a given theoretical method for the calculation of the absorption spectra, not only the energy of the LSP peak has to be considered, but also the oscillator strength $f_{\text {osc }}$ associated with it. The oscillator strength is readily available in an eigenvalue formulation of QHT [60,120]. Our QHT implementation is frequency dependent, and, therefore, the oscillator strength is not directly computed, but it can be extracted from the absorption spectra using the fitting procedure described in Sec. IV of the Supplemental Material [110]. The oscillator strength of the LSP peak can also be extracted from the TD-DFT spectra if the onset of the plasmon shoulder is considered (Sec. IV of the Supplemental 
Material [110]). Previous attempts to compute the $f_{\text {osc }}$ of the LSP peak are based on the sum-rule approaches [91]. In Fig. 10(b), we report $f_{\text {osc }}$ of the LSP peak, as obtained from TD-DFT and the same KE functionals. Figure 10(b) shows that for all methods, LSP converges to the classical Mie results for large $N_{e}$. However, $f_{\text {osc }}$ for $\mathrm{KS} / \mathrm{TFvW}$ and $\mathrm{KS} /$ PGS is largely underestimated, as the main plasmon peak is subdivided into different peaks, as previously discussed. On the other hand, the main peak of KS/PGSL contains almost all the oscillator strength, as in the classical calculations. The mean average error with respect to reference TD-DFT is reported in the second part of Table I. QHT-TFvW and QHT-PGS are thus quite inaccurate for the oscillator strength, whereas PGSL has an error of less than 3\%. In all cases, better accuracy is obtained using the model density. Thus, while QHT-TFvW (QHTPGS) and QHT-PGSL give either very good LSP energy or very good LSP oscillator strength, QHT-PGSLN is the only functional which gives very good accuracy for both properties.

Finally, we consider the Feibelman $d$-parameter [106], i.e.,

$$
d=\frac{\int 4 \pi r^{2}(r-R) n_{1} d r}{\int 4 \pi r^{2} n_{1} d r}
$$

where $R$ is the radius of the jellium nanosphere, and $n_{1}$ is the radial part of the induced density. Equation (25) is valid for a spherical density, and the real part of $d$ describes the position of the center of mass of $n_{1}$ with respect $R$. The results are reported in Fig. 10(c) and in the last section of Table I. While for PGSL the $d$-parameter is underestimated (i.e., the induced density is more confined inside the nanoparticles), TFvW, PGS, and PGSLN give quite accurate results, as also shown in Fig. 9.

The PGSLN is thus simultaneously very accurate for the LSP energy position, Bennett energy position, LSP oscillator strength, and Feibelman $d$-parameter for all the systems considered. This is quite a large test set of properties and systems, showing the reliability of the $q_{0}$ parameter and of the PGSLN functional form.

\section{APPLICATION TO SPHERICAL DIMER}

Our FEM implementation allows us to calculate absorption spectra for axisymmetric structures. An important example of such a system is a nanosphere dimer. The NP dimer has been widely studied in the literature since it supports gap plasmons that can squeeze light down to subnanometer volumes, making it an ideal system for exploring the quantum and nonlocal phenomena [21,70, 121-123]. Here we consider a dimer of $\mathrm{Na}$ jellium spheres with 1074 electrons each. In Fig. 11(a), we present a comparison of the absorption cross section as calculated from the Mod/TFvW, Mod/PGSL, and Mod/PGSLN (the cross section is normalized to the $2 \sigma_{0}=\pi R^{2}$ with $R$ being
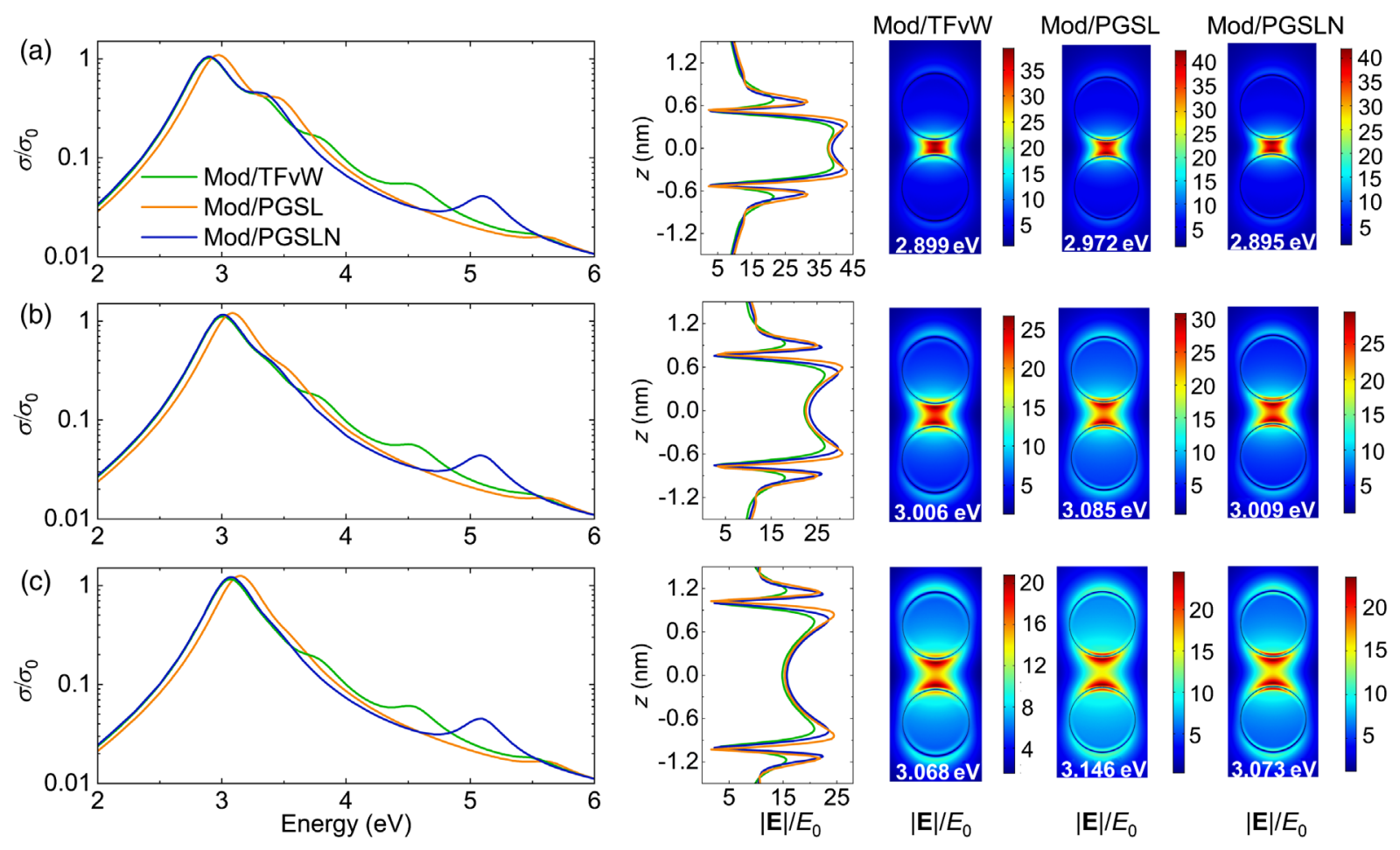

FIG. 11. Normalized absorption cross section $\sigma / \sigma_{0}$ (in logarithmic scale) and norm of the total field $|\mathbf{E}| / E_{0}$ for dimers of Na jellium spheres with $N_{e}=1074$ electrons as obtained from the Mod/TFvW, Mod/PGS, and Mod/PGSL. From top to bottom: panel (a) refers to gap $=1 \mathrm{~nm}$, (b) to gap $=1.5 \mathrm{~nm}$, and (c) refers to gap $=2 \mathrm{~nm}$. 
TABLE II. The average value of $|\mathbf{E}| / E_{0}$ in the dimer gap as calculated from QHT with different KE functionals (TFvW, PGS, PGSL, and PGSLN) and the model density.

\begin{tabular}{lcc}
\hline \hline Method & Gap & $|\mathbf{E}| / E_{0}$ \\
\hline Mod/TFvW & $1.0 \mathrm{~nm}$ & 33.9 \\
& $1.5 \mathrm{~nm}$ & 22.5 \\
Mod/PGS & $2.0 \mathrm{~nm}$ & 16.5 \\
& $1.0 \mathrm{~nm}$ & 35.4 \\
& $1.5 \mathrm{~nm}$ & 23.2 \\
Mod/PGSL & $2.0 \mathrm{~nm}$ & 16.9 \\
& $1.0 \mathrm{~nm}$ & 38.6 \\
& $1.5 \mathrm{~nm}$ & 25.4 \\
Mod/PGSLN & $2.0 \mathrm{~nm}$ & 18.7 \\
& $1.0 \mathrm{~nm}$ & 36.8 \\
& $1.5 \mathrm{~nm}$ & 24.6 \\
\hline \hline
\end{tabular}

the radius of a single sphere). The plane wave that excites the structure is polarized along the $z$ axis, and the input groundstate density is the sum of model densities [see Eq. (21)] of two spheres. As we can see, the Mod/TFvW gives oscillations in the spectrum (at approximately 3.9 and $4.6 \mathrm{eV}$ ), which are absent in the Mod/PGSL and Mod/PGSLN approaches. Our convergence analysis shows that these oscillations, as in the case of the sphere (see Fig. 3), persist and depend on the computational domain. These oscillations should not be confused with the small undulation next to the main plasmon peak that is more clearly visible in the gap $=1 \mathrm{~nm}$ case [Fig. 11(a)]. This undulation comes from higher-order plasmon resonances and gets higher for smaller sizes of the gap [21]. For all considered cases of gap size, Mod/PGSL gives blueshifted plasmon resonance energy with respect to other methods. On the other hand, Mod/ TFvW and Mod/PGSLN match very well at the plasmon resonance, with the maximum difference of $0.005 \mathrm{eV}$, but, as stated before, Mod/PGSLN does not show the oscillations. Also, the Bennett peak is observed in the Mod/PGSL (approximately $5.7 \mathrm{eV}$ ), and Mod/PGSLN (approximately $5.1 \mathrm{eV}$ ) approaches that is stable to change in the computational domain size. The respective values of the plasmon resonance are shown in the map plots of the total field enhancement in Fig. 11. There we also see that the field gets more enhanced for Mod/QHT-PGSL as it does for other approaches, which is more clearly observed in the cut lines of the field distribution around the $z$ axis. This behavior is expected since Mod/PGSL does not result in additional peaks of the absorption spectra as opposed to TFvW, and now more energy is moved to the main plasmon peak. For Mod/ PGSLN, the Bennett peak is more pronounced as opposed to Mod/PGSL, and, consequently, we have less enhancement of PGSLN calculations at the main plasmon peak. Also, as Table II shows, more field is concentrated in the gap for Mod/QHT-PGSL.

\section{CONCLUSIONS AND FUTURE PERSPECTIVES}

We extend the quantum hydrodynamic theory to Laplacian-level kinetic energy functionals. In particular, we start our investigation considering the PGSL functional, which is shown to be accurate for OF-DFT calculations of metals and semiconductors [97]. We analyze in detail $\mathrm{Na}$ jellium nanospheres, and the results are compared to gradient-level kinetic functional and reference TD-DFT calculations. The key results obtained are focused on two main findings:

(i) QHT-TFvW and QHT-PGS that are based on gradient-level KE functionals of electron density, together with an LSP resonance, give additional resonances in the absorption spectrum of $\mathrm{Na}$ jellium nanospheres. These resonances have usually an energy higher than the critical frequency, and thus, they are very sensitive to the computational domain size. Well-defined additional resonances are not present in TD-DFT or in QHT-TFvW with an infinite computational domain size. In both cases, only a shoulder is present at the high-energy side to the plasmon peak, with the TD-DFT result being much smaller and at higher energy than in QHT-TFvW. On the other hand, QHT-PGSL yields only the LSP peak in the absorption spectrum, with an overall spectrum and oscillator strength closer to TD-DFT.

(ii) The theoretical and numerical asymptotic analysis of the induced charge density as obtained from QHTTFvW and QHT-PGS shows that the decay slope is changing at different energies of incident radiation. Contrarily, QHT-PGSL shows the same decay slope for all energies, and thus, no critical frequency exists anymore. This result strongly simplifies the boundary conditions so that a converged calculation can be obtained with a very small computational domain size.

Our results thus demonstrate that the convergence of the QHTabsorption spectra is problematic, and most of the QHT$\mathrm{TFvW}$ results reported so far are thus not accurate enough for energies above LSP resonance. The QHT-PGSL, on the other hand, does not suffer from these problems.

The PGSL functional, which is characterized by a term proportional to $q^{2}$, solves some fundamental limitation of the QHT-TFvW approaches: (i) the presence of the critical frequency, (ii) the sensitivity to input density and the computational domain size, and (iii) the underestimation of the oscillator strength for the LSP peak. On the other hand, the PGSL results are not very accurate when the LSP energy position and LSP $d$-parameter are considered. Moreover, PGSL predicts a Bennett state too close to the volume plasmon.

We find that all these shortcomings can be removed if the $q^{2}$ term is kept only outside the nanoparticles. We thus develop a new functional, PGSLN, which combines only 
the good features of QHT-TFvW (or QHT-PGS) and QHTPGSL. Thus, QHT-PGSLN is very accurate for all properties that are of interest in plasmonics, allowing an efficient and numerically converged computation of collective excitations in quantum systems. Clearly, the PGSLN functional is a very simple functional introduced here to show the power of the Laplacian-level QHT, but further tests and developments will be required to verify and extend its applicability.

The Laplacian-level QHT is thus a new platform, very promising for the future, as the Laplacian ingredient includes many more degrees of freedom in developing accurate KE functionals than a more conventional functional based on the density gradient. So far, however, the development of a semilocal KE functional focused only on ground-state properties, considering only the total $\mathrm{KE}$ and the KE potential (i.e., the first functional derivatives). Instead, for the QHT response properties, the KE kernel (i.e., the second functional derivative) is required, but, so far, it has not been considered at all in the semilocal KE functional development $[97,99,100,102-105]$.

In addition, it is crucial to understand the role of static and dynamic corrections to the energy functional. Although here we consider only static corrections at the second-order gradient and Laplacian level, the analysis of dynamic correction represents another important route to explore. In particular, a frequency-dependent damping can be important to further improve the accuracy of QHTPGSLN with respect to TD-DFT. Overall, we believe that our current results will help to better understand the role of functional dependence on electron density in plasmonic systems.

\section{ACKNOWLEDGMENTS}

H. B. and C. C. conceived the idea of applying the PGSL functional to QHT. H. B. derived the first-order potential and implemented all the equations in FEM. H. B. performed calculations for the dimer. H. B. and F. D. S. did the calculations for the nanospheres with different numbers of electrons. F.D.S. derived the proof for the asymptotic decay and the PGSLN functional. F.D. S. and C. C. supervised the research.

All authors contributed to writing the paper. H. M. B. and F. D. S. contributed equally to this work.

\section{APPENDIX A: ABSORPTION SPECTRUM}

In QHT, the absorption cross section is calculated as

$$
\sigma(\omega)=\frac{\omega}{2 I_{0}} \int \operatorname{Im}\left\{\mathbf{E} \cdot \mathbf{P}^{*}\right\} d V,
$$

with $I_{0}$ being the intensity for the incident plane wave with frequency $\omega$. The electric field $\mathbf{E}$ and the polarization vector $\mathbf{P}$ are obtained solving Eqs. (2a) and (2b). Considering the very small size of the investigated nanoparticles, only dipole modes are excited (for spherical nanoparticles). An important parameter for the shape of the absorption spectra is the damping parameter $(\gamma)$; see Eq. (2b). If not stated differently, in all QHT calculations, we use $\gamma=66 \mathrm{meV}$.

The normalized absorption cross section (absorption efficiency) is then obtained by normalizing $\sigma$ to the geometric cross section of a nanosphere $\sigma_{0}=\pi R^{2}$ with $R$ being the radius of the nanosphere.

The TD-DFT absorption spectra are computed with a finite-difference in-house code (with spherical symmetry) introduced in Ref. [62]; a radial uniform grid is used to represent KS orbitals and densities. In TD-DFT, no retardation effects are included, and only longitudinal electric fields are considered [19]. The absorption cross section [124-127] is calculated as

$$
\sigma(\omega)=\frac{\omega}{c \epsilon_{0}} \operatorname{Im}\left\{\alpha_{z z}(\omega)\right\},
$$

where the polarizability is given by

$$
\alpha_{z z}(\omega)=-e^{2} \int d \mathbf{r} d \mathbf{r}^{\prime} z \chi\left(\mathbf{r}, \mathbf{r}^{\prime}, \omega\right) z^{\prime}
$$

with $\chi\left(\mathbf{r}, \mathbf{r}^{\prime}, \omega\right)=\delta n(\mathbf{r}) / \delta\left(e V_{\text {ext }}\left(\mathbf{r}^{\prime}\right)\right)$ being the interacting density-density response function [19], which is obtained solving the Dyson equation

$$
\chi=\chi_{0}+\chi_{0}\left(v_{\mathrm{Coul}}+f_{\mathrm{XC}}^{\mathrm{LDA}}\right) \chi .
$$

In Eq. (A4), $v_{\mathrm{Coul}}$ is the Coulomb interaction, $f_{\mathrm{XC}}^{\mathrm{LDA}}$ is the adiabatic LDA XC kernel, and $\chi_{0}$ is the noninteracting density-density response function, which is computed using the Green's function [125] using occupied KS orbitals from the ground-state calculation (again using LDA). The broadening parameter for the Green's function calculations is, if not stated differently, $\Gamma_{0}=33 \mathrm{meV}$.

\section{APPENDIX B: FEM IMPLEMENTATION}

In order to lower the order of derivatives, we multiply Eq. (2b) by test function $\tilde{\mathbf{P}}$ and integrate by parts, which give us

$$
\begin{aligned}
& \int\left\{-\frac{e}{m_{e}}\left(\frac{\delta G[n]}{\delta n}\right)_{1}(\nabla \cdot \tilde{\mathbf{P}})+\frac{1}{n_{0}}\left[\left(\omega^{2}+i \gamma \omega\right) \mathbf{P}+\epsilon_{0} \omega_{p}^{2} \mathbf{E}\right] \cdot \tilde{\mathbf{P}}\right\} \\
& d V=0,
\end{aligned}
$$

where we assume that the integral on the boundary goes to zero. Even after integration by parts, the $(\delta G[n] / \delta n)_{1}$ potential contains derivatives up to the fourth order of $n_{1}$ [see the Eqs. (12)], so auxiliary variables should be added to lower the order of differentiation. By introducing two variables $\mathbf{F}=\nabla n_{1}$ and $\mathbf{O}=\nabla\left(\nabla^{2} n_{1}\right)=\nabla^{2} \mathbf{F}$, we 
have only first-order derivatives. Considering axisymmetry of considered structures, we adopt the 2.5D technique $[62,113,114]$, and the dependence of $\mathbf{E}, \mathbf{P}, \mathbf{F}$, and $\mathbf{O}$ on the azimuthal coordinate is taken in $e^{-i m \phi}$ form with $m \in \mathbb{Z}$. The dependence on $m$ for test functions $\tilde{\mathbf{E}}, \tilde{\mathbf{P}}, \tilde{\mathbf{F}}$, and $\tilde{\mathbf{O}}$ is of $e^{i m \phi}$ form. Thus, instead of a three-dimensional problem, we can have $2 m_{\max }+1$ problems (with $m_{\max }$ being the maximum value for $m$ ). Moreover, for the dimensions considered in the current work $m_{\max }=0$ is enough for the convergence of results. Finally, only one two-dimensional problem needs to be solved. Hence, we come to the following system of equations:

$$
\begin{gathered}
2 \pi \int\left\{\left(\nabla \times \mathbf{E}^{(0)}\right)\left(\nabla \times \tilde{\mathbf{E}}^{(0)}\right)-\left[k_{0}^{2} \mathbf{E}(0)+\mu_{0} \omega^{2} \mathbf{P}^{(0)}\right] \cdot \tilde{\mathbf{E}}^{(0)}\right\} \rho d \rho d z=0, \\
2 \pi \int\left\{-\frac{e}{m_{e}}\left(\frac{\delta G[n]}{\delta n}\right)_{1}^{(0)}\left(\nabla \cdot \tilde{\mathbf{P}}^{(0)}\right)+\frac{1}{n_{0}}\left[\left(\omega^{2}+i \gamma \omega\right) \mathbf{P}^{(0)}+\epsilon_{0} \omega_{p}^{2}\left(\mathbf{E}^{(0)}+\mathbf{E}_{\mathrm{inc}}^{(0)}\right)\right] \cdot \tilde{\mathbf{P}}^{(0)}\right\} \rho d \rho d z=0, \\
2 \pi \int\left\{\left(\nabla \cdot \mathbf{P}^{(0)}\right)\left(\nabla \cdot \tilde{\mathbf{F}}^{(0)}\right)+e \mathbf{F}^{(0)} \cdot \tilde{\mathbf{F}}^{(0)}\right\} \rho d \rho d z=0, \\
2 \pi \int\left\{\left(\nabla \cdot \mathbf{F}^{(0)}\right)\left(\nabla \cdot \tilde{\mathbf{O}}^{(0)}\right)+\mathbf{O}^{(0)} \cdot \tilde{\mathbf{O}}^{(0)}\right\} \rho d \rho d z=0,
\end{gathered}
$$

where the (0) superscript denotes the zero-order coefficients of the $\mathbf{v}(\rho, \phi, z)=\sum_{m \in \mathbb{Z}} \mathbf{v}^{(m)}(\rho, z) e^{-i m \phi}$ vector field expansion of cylindrical harmonics. We find that curl elements for Eq. (B2a) and divergence elements [112] for other equations of the system (B2) are the best choices for stable solutions.

For the wave equation (B2), simulation domain radius $R_{\text {dom }}$ is defined via the $R_{\text {dom }}=R+500 a_{0}$ condition. $R=$ $r_{s} N_{e}^{1 / 3}$ is the radius of the nanosphere, and, for dimers, it is the radius of one of the spheres. Perfectly matched layers (PMLs) are used in order to emulate an infinite domain and avoid unwanted reflections. The PML thickness is set to $t_{\mathrm{PML}}=200 a_{0}$ for all the considered systems. Also, a zero flux boundary condition is imposed on the electric field at the outer boundary of the PML. For Eqs. (B2b)-(B2d), simulations are done in a smaller domain, considering faster decay of variables $\mathbf{P}, \mathbf{F}$, and $\mathbf{O}$ compared to the electric field. The domain, as depicted in Fig. S1(a) of the Supplemental Material [110], is a semicircle (consider the axial symmetry) for the nanospheres, and for the dimers it is the union of two circles centered at the centers of the nanospheres. Moreover, to facilitate the calculations, only the "quarter" of the dimer is simulated with a corresponding perfect electric conductor condition at the intersection segment of two circles, as shown in Fig. S1(b) of the Supplemental Material [110]. The radius for the circles is $r_{\mathrm{dom}}=R+25 a_{0}$ for QHT and QHT-PGS, but for QHTPGSL, it is in the range $r_{\mathrm{dom}} \approx R+12 a_{0}$. The simulation domain is smaller for QHT-PGSL because the $\nu \approx 1.12$ decay slope is bigger in this case (see Sec. VI). Dirichlet boundary conditions $\mathbf{P}=0, \mathbf{F}=0$, and $\mathbf{O}=0$ are set on the simulation domain boundary. As we state in Sec. VI, mixed boundary condition with a fixed exponential decay can be used for QHT-PGSL, so that a very small simulation domain is enough for converged calculations.

[1] Modern Plasmonics, Handbook of Surface Science Vol. 4, edited by A. A. Maradudin, J. R. Sambles, and W. L. Barnes (Elsevier, Amsterdam, 2014).

[2] M. Kauranen and A. V. Zayats, Nonlinear Plasmonics, Nat. Photonics 6, 737 (2012).

[3] M. Ren, E. Plum, J. Xu, and N. I. Zheludev, Giant Nonlinear Optical Activity in a Plasmonic Metamaterial, Nat. Commun. 3, 833 (2012).

[4] M. L. Brongersma, N. J. Halas, and P. Nordlander, Plasmon-Induced Hot Carrier Science and Technology, Nat. Nanotechnol. 10, 25 (2015).

[5] I. Goykhman, B. Desiatov, J. Khurgin, J. Shappir, and U. Levy, Locally Oxidized Silicon Surface-Plasmon Schottky Detector for Telecom Regime, Nano Lett. 11, 2219 (2011).

[6] H. Xu, E. J. Bjerneld, M. Käll, and L. Börjesson, Spectroscopy of Single Hemoglobin Molecules by Surface Enhanced Raman Scattering, Phys. Rev. Lett. 83, 4357 (1999).

[7] S. Kawata, Y. Inouye, and P. Verma, Plasmonics for NearField Nano-Imaging and Superlensing, Nat. Photonics 3, 388 (2009).

[8] J. Weiner and F. Nunes, Light-Matter Interaction: Physics and Engineering at the Nanoscale, 2nd ed. (Oxford University Press, New York, 2017).

[9] C. F. Bohren and D. R. Huffman, Absorption and Scattering of Light by Small Particles (Wiley-VCH, Weinheim, 2004).

[10] M. Quinten, Optical Properties of Nanoparticle Systems: Mie and Beyond (Wiley-VCH, Weinheim, 2011). 
[11] S. M. Morton, D. W. Silverstein, and L. Jensen, Theoretical Studies of Plasmonics Using Electronic Structure Methods, Chem. Rev. 111, 3962 (2011).

[12] R. Esteban, A. G. Borisov, P. Nordlander, and J. Aizpurua, Bridging Quantum and Classical Plasmonics with a Quantum-Corrected Model, Nat. Commun. 3, 825 (2012).

[13] Y. Luo, A. I. Fernandez-Dominguez, A. Wiener, S. A. Maier, and J. B. Pendry, Surface Plasmons and Nonlocality: A Simple Model, Phys. Rev. Lett. 111, 093901 (2013).

[14] W. Yan, M. Wubs, and N. A. Mortensen, Projected Dipole Model for Quantum Plasmonics, Phys. Rev. Lett. 115, 137403 (2015).

[15] S. Raza, S. I. Bozhevolnyi, M. Wubs, and N. A. Mortensen, Nonlocal Optical Response in Metallic Nanostructures, J. Phys. Condens. Matter 27, 183204 (2015).

[16] A. Varas, P. García-González, J. Feist, F. García-Vidal, and A. Rubio, Quantum Plasmonics: From Jellium Models to Ab Initio Calculations, Nanophotonics 5, 409 (2016).

[17] T. Christensen, W. Yan, A.-P. Jauho, M. Soljačić, and N. A. Mortensen, Quantum Corrections in Nanoplasmonics: Shape, Scale, and Material, Phys. Rev. Lett. 118, 157402 (2017).

[18] E. Runge and E. K. U. Gross, Density-Functional Theory for Time-Dependent Systems, Phys. Rev. Lett. 52, 997 (1984).

[19] C. A. Ullrich, Time-Dependent Density-Functional Theory: Concepts and Applications, Oxford Graduate Texts (Oxford University Press, New York, 2012).

[20] P. Zhang, J. Feist, A. Rubio, P. García-González, and F. J. García-Vidal, Ab Initio Nanoplasmonics: The Impact of Atomic Structure, Phys. Rev. B 90, 161407(R) (2014).

[21] M. Barbry, P. Koval, F. Marchesin, R. Esteban, A. G. Borisov, J. Aizpurua, and D. Sánchez-Portal, Atomistic Near-Field Nanoplasmonics: Reaching Atomic-Scale Resolution in Nanooptics, Nano Lett. 15, 3410 (2015).

[22] R. Sinha-Roy, P. García-González, H.-C. Weissker, F. Rabilloud, and A. I. Fernández-Domínguez, Classical and Ab Initio Plasmonics Meet at Sub-Nanometric Noble Metal Rods, ACS Photonics 4, 1484 (2017).

[23] P. Zhang, W. Jin, and W. Liang, Size-Dependent Optical Properties of Aluminum Nanoparticles: From Classical to Quantum Description, J. Phys. Chem. C 122, 10545 (2018).

[24] F. Della Sala, M. Pezzolla, S. D’Agostino, and E. Fabiano, Ab Initio Plasmonics of Externally Doped Silicon Nanocrystals, ACS Photonics 6, 1474 (2019).

[25] E. Madelung, Quantentheorie in Hydrodynamischer Form, Z. Phys. 40, 322 (1927).

[26] F. Bloch, Bremsvermögen von Atomen mit Mehreren Elektronen, Z. Phys. 81, 363 (1933).

[27] H. Jensen, Eigenschwingungen eines Fermi-Gases und Anwendung auf die Blochsche Bremsformel für Schnelle Teilchen, Z. Phys. 106, 620 (1937).

[28] S. C. Ying, Hydrodynamic Response of Inhomogeneous Metallic Systems, Nuovo Cimento Soc. Ital. Fis. 23B, 270 (1974).

[29] R. G. Parr and W. Yang, Density-Functional Theory of Atoms and Molecules, International Series of Monographs on Chemistry (Oxford University Press, New York, 1994).
[30] Y. A. Wang, N. Govind, and E. A. Carter, Orbital-Free Kinetic-Energy Functionals for the Nearly Free Electron Gas, Phys. Rev. B 58, 13465 (1998).

[31] Recent Progress in Orbital-free Density Functional Theory, edited by T. A. Wesolowski and Y. A. Wang (World Scientific, Singapore, 2013).

[32] L. H. Thomas, The Calculation of Atomic Fields, Math. Proc. Cambridge Philos. Soc. 23, 542 (1927).

[33] E. Fermi, Un Metodo Statistico per la Determinazione di alcune Prioprietà dell'Atomo, Rend. Accad. Naz. Lincei 6, 602 (1927), http://operedigitali.lincei.it/Fermi/Fermiomnia1_4_178-385.pdf.

[34] V. Gavini, K. Bhattacharya, and M. Ortiz, Quasi-Continuum Orbital-Free Density-Functional Theory: A Route to Multi-Million Atom Non-Periodic DFT Calculation, J. Mech. Phys. Solids 55, 697 (2007).

[35] J. Xia and E. A. Carter, Density-Decomposed OrbitalFree Density Functional Theory for Covalently Bonded Molecules and Materials, Phys. Rev. B 86, 235109 (2012).

[36] I. Shin and E. A. Carter, Enhanced von Weizsäcker WangGovind-Carter Kinetic Energy Density Functional for Semiconductors, J. Chem. Phys. 140, 18A531 (2014).

[37] L. A. Constantin, E. Fabiano, and F. Della Sala, Nonlocal Kinetic Energy Functional from the Jellium-with-Gap Model: Applications to Orbital-Free Density Functional Theory, Phys. Rev. B 97, 205137 (2018).

[38] W. C. Witt, B. G. del Rio, J. M. Dieterich, and E. A. Carter, Orbital-Free Density Functional Theory for Materials Research, J. Mater. Res. 33, 777 (2018).

[39] A. Domps, P.-G. Reinhard, and E. Suraud, Time-Dependent Thomas-Fermi Approach for Electron Dynamics in Metal Clusters, Phys. Rev. Lett. 80, 5520 (1998).

[40] D. Neuhauser, S. Pistinner, A. Coomar, X. Zhang, and G. Lu, Dynamic Kinetic Energy Potential for Orbital-Free Density Functional Theory, J. Chem. Phys. 134, 144101 (2011).

[41] H. Xiang, M. Zhang, X. Zhang, and G. Lu, Understanding Quantum Plasmonics from Time-Dependent Orbital-Free Density Functional Theory, J. Phys. Chem. C 120, 14330 (2016).

[42] X. Zhang, H. Xiang, M. Zhang, and G. Lu, Plasmonic Resonances of Nanoparticles from Large-Scale Quantum Mechanical Simulations, Int. J. Mod. Phys. B 31, 1740003 (2017).

[43] N. W. Ashcroft and N. D. Mermin, Solid State Physics (Holt, Rinehart and Winston, New York, 1976).

[44] C. Ciracì, R. T. Hill, J. J. Mock, Y. Urzhumov, A. I. Fernandez-Dominguez, S. A. Maier, J. B. Pendry, A. Chilkoti, and D. R. Smith, Probing the Ultimate Limits of Plasmonic Enhancement, Science 337, 1072 (2012).

[45] S. Raza, N. Stenger, S. Kadkhodazadeh, S. V. Fischer, N. Kostesha, A.-P. Jauho, A. Burrows, M. Wubs, and N. A. Mortensen, Blueshift of the Surface Plasmon Resonance in Silver Nanoparticles Studied with EELS, Nanophotonics 2 , 131 (2013).

[46] T. V. Teperik, P. Nordlander, J. Aizpurua, and A. G. Borisov, Robust Subnanometric Plasmon Ruler by Rescaling of the Nonlocal Optical Response, Phys. Rev. Lett. 110, 263901 (2013). 
[47] T. Reiners, C. Ellert, M. Schmidt, and H. Haberland, Size Dependence of the Optical Response of Spherical Sodium Clusters, Phys. Rev. Lett. 74, 1558 (1995).

[48] J.-H. Li, M. Hayashi, and G.-Y. Guo, Plasmonic Excitations in Quantum-Sized Sodium Nanoparticles Studied by Time-Dependent Density Functional Calculations, Phys. Rev. B 88, 155437 (2013).

[49] C. David and F. J. G. de Abajo, Surface Plasmon Dependence on the Electron Density Profile at Metal Surfaces, ACS Nano 8, 9558 (2014).

[50] C. F. von Weizsäcker, Zur Theorie der Kernmassen, Z. Phys. 96, 431 (1935).

[51] G. Manfredi, How to Model Quantum Plasma, Fields Inst. Commun. 46, 263 (2005).

[52] Complex Plasmas: Scientific Challenges and Technological Opportunities, Springer Series on Atomic, Optical, and Plasma Physics Vol. 82, edited by M. Bonitz, J. Lopez, K. Becker, and H. Thomsen (Springer International Publishing, Cham, 2014).

[53] P. K. Shukla and B. Eliasson, Novel Attractive Force between Ions in Quantum Plasmas, Phys. Rev. Lett. 108, 165007 (2012).

[54] D. Michta, F. Graziani, and M. Bonitz, Quantum Hydrodynamics for Plasmas-A Thomas-Fermi Theory Perspective, Contrib. Plasma Phys. 55, 437 (2015).

[55] Z. A. Moldabekov, M. Bonitz, and T. S. Ramazanov, Theoretical Foundations of Quantum Hydrodynamics for Plasmas, Phys. Plasmas 25, 031903 (2018).

[56] M. Bonitz, Z. A. Moldabekov, and T.S. Ramazanov, Quantum Hydrodynamics for Plasmas-Quo vadis?, Phys. Plasmas 26, 090601 (2019).

[57] E. Zaremba and H. C. Tso, Thomas-Fermi-Dirac-von Weizsäcker Hydrodynamics in Parabolic Wells, Phys. Rev. B 49, 8147 (1994).

[58] B. P. van Zyl and E. Zaremba, Thomas-Fermi-Dirac-von Weizsäcker Hydrodynamics in Laterally Modulated Electronic Systems, Phys. Rev. B 59, 2079 (1999).

[59] E. Zaremba and H. Tso, in Electronic Density Functional Theory, edited by J. Dobson, G. Vignale, and M. Das (Springer, Boston, 1998), pp. 227-242.

[60] A. Banerjee and M. K. Harbola, Hydrodynamical Approach to Collective Oscillations in Metal Clusters, Phys. Lett. A 372, 2881 (2008).

[61] G. Toscano, J. Straubel, A. Kwiatkowski, C. Rockstuhl, F. Evers, H. Xu, N. Asger Mortensen, and M. Wubs, Resonance Shifts and Spill-Out Effects in Self-Consistent Hydrodynamic Nanoplasmonics, Nat. Commun. 6, 7132 (2015).

[62] C. Ciracì and F. Della Sala, Quantum Hydrodynamic Theory for Plasmonics: Impact of the Electron Density Tail, Phys. Rev. B 93, 205405 (2016).

[63] K. Ding and C. T. Chan, Plasmonic Modes of Polygonal Rods Calculated Using a Quantum Hydrodynamics Method, Phys. Rev. B 96, 125134 (2017).

[64] K. Ding and C. T. Chan, Optical Forces, Torques, and Force Densities Calculated at a Microscopic Level Using a Self-Consistent Hydrodynamics Method, Phys. Rev. B 97, 155118 (2018).

[65] M. Khalid, F. Della Sala, and C. Ciracì, Optical Properties of Plasmonic Core-Shell Nanomatryoshkas: A Quantum Hydrodynamic Analysis, Opt. Express 26, 17322 (2018).
[66] M. Khalid and C. Ciracì, Numerical Analysis of Nonlocal Optical Response of Metallic Nanoshells, Photonics 6, 39 (2019).

[67] Y.-Y. Zhang, S.-B. An, Y.-H. Song, N. Kang, Z. L. Mikovi, and Y.-N. Wang, Plasmon Excitation in Metal Slab by Fast Point Charge: The Role of Additional Boundary Conditions in Quantum Hydrodynamic Model, Phys. Plasmas 21, 102114 (2014).

[68] W. Yan, Hydrodynamic Theory for Quantum Plasmonics: Linear-Response Dynamics of the Inhomogeneous Electron Gas, Phys. Rev. B 91, 115416 (2015).

[69] D. I. Palade, Multiple Surface Plasmons in an Unbounded Quantum Plasma Half-Space, Phys. Plasmas 23, 074504 (2016).

[70] C. Ciracì, Current-Dependent Potential for Nonlocal Absorption in Quantum Hydrodynamic Theory, Phys. Rev. B 95, 245434 (2017).

[71] C. Ciracì, R. Jurga, M. Khalid, and F. Della Sala, Plasmonic Quantum Effects on Single-Emitter Strong Coupling, Nanophotonics 8, 1821 (2019).

[72] D. de Ceglia, M. Scalora, M. A. Vincenti, S. Campione, K. Kelley, E. L. Runnerstrom, J.-P. Maria, G. A. Keeler, and T. S. Luk, Viscoelastic Optical Nonlocality of Low-Loss Epsilon-near-Zero Nanofilms, Sci. Rep. 8, 9335 (2018).

[73] A. Bergara, J. Pitarke, and R. Ritchie, Nonlinear Quantum Hydrodynamical Model of the Electron Gas, Nucl. Instrum. Methods Phys. Res., Sect. B 115, 70 (1996).

[74] N. Crouseilles, P.-A. Hervieux, and G. Manfredi, Quantum Hydrodynamic Model for the Nonlinear Electron Dynamics in Thin Metal Films, Phys. Rev. B 78, 155412 (2008).

[75] M. Khalid and C. Ciracì, Enhancing second-harmonic generation with electron spill-out at metallic surfaces, Communications in Physics 3, 214 (2020).

[76] A. J. Bennett, Influence of the Electron Charge Distribution on Surface-Plasmon Dispersion, Phys. Rev. B 1, 203 (1970).

[77] A. Eguiluz, S. Ying, and J. Quinn, Influence of the Electron Density Profile on Surface Plasmons in a Hydrodynamic Model, Phys. Rev. B 11, 2118 (1975).

[78] C. Schwartz and W. L. Schaich, Hydrodynamic Models of Surface Plasmons, Phys. Rev. B 26, 7008 (1982).

[79] A. Moradi, Quantum Nonlocal Effects on Optical Properties of Spherical Nanoparticles, Phys. Plasmas 22, 022119 (2015).

[80] M. Kupresak, X. Zheng, G. A. E. Vandenbosch, and V. V. Moshchalkov, Appropriate Nonlocal Hydrodynamic Models for the Characterization of Deep-Nanometer Scale Plasmonic Scatterers, Adv. Theory Simul. 3, 1900172 (2019).

[81] A. Moradi, Canonical Problems in the Theory of Plasmonics, Springer Series in Optical Sciences Vol. 230 (Springer International Publishing, Cham, 2020).

[82] R. Ruppin, Optical Properties of a Plasma Sphere, Phys. Rev. Lett. 31, 1434 (1973).

[83] B. B. Dasgupta and R. Fuchs, Polarizability of a Small Sphere Including Nonlocal Effects, Phys. Rev. B 24, 554 (1981).

[84] S. Raza, G. Toscano, A.-P. Jauho, M. Wubs, and N. A. Mortensen, Unusual Resonances in Nanoplasmonic Structures due to Nonlocal Response, Phys. Rev. B 84, 121412(R) (2011). 
[85] K.-D. Tsuei, E. W. Plummer, A. Liebsch, K. Kempa, and P. Bakshi, Multipole Plasmon Modes at a Metal Surface, Phys. Rev. Lett. 64, 44 (1990).

[86] K.-D. Tsuei, E. Plummer, A. Liebsch, E. Pehlke, K. Kempa, and P. Bakshi, The Normal Modes at the Surface of Simple Metals, Surf. Sci. 247, 302 (1991).

[87] A. Liebsch, Electronic Excitations at Metal Surfaces (Springer, New York, 1997).

[88] D. E. Beck, Self-Consistent Calculation of the Eigenfrequencies for the Electronic Excitations in Small Jellium Spheres, Phys. Rev. B 35, 7325 (1987).

[89] M. Brack, The Physics of Simple Metal Clusters: SelfConsistent Jellium Model and Semiclassical Approaches, Rev. Mod. Phys. 65, 677 (1993).

[90] C. Yannouleas, E. Vigezzi, and R. A. Broglia, Evolution of the Optical Properties of Alkali-Metal Microclusters towards the Bulk: The Matrix Random-Phase-Approximation Description, Phys. Rev. B 47, 9849 (1993).

[91] P.-G. Reinhard, O. Genzken, and M. Brack, From Sum Rules to RPA: 3. Optical Dipole Response in Metal Clusters, Ann. Phys. (N.Y.) 508, 576 (1996).

[92] A. Liebsch, Surface-Plasmon Dispersion and Size Dependence of Mie Resonance: Silver versus Simple Metals, Phys. Rev. B 48, 11317 (1993).

[93] M. K. Harbola, Differential Virial Theorem and Quantum Fluid Dynamics, Phys. Rev. A 58, 1779 (1998).

[94] M. Brack, B. Jennings, and Y. Chu, On the Extended Thomas-Fermi Approximation to the Kinetic Energy Density, Phys. Lett. 65B, 1 (1976).

[95] D. I. Palade, Nonlocal Orbital-Free Kinetic Pressure Tensors for the Fermi Gas, Phys. Rev. B 98, 245401 (2018).

[96] Y. A. Wang and E. A. Carter, in Theoretical Methods in Condensed Phase Chemistry Vol. 5, edited by S.D. Schwartz (Kluwer Academic Publishers, Dordrecht, 2002), pp. 117-184.

[97] L. A. Constantin, E. Fabiano, and F. Della Sala, Semilocal Pauli-Gaussian Kinetic Functionals for Orbital-Free Density Functional Theory Calculations of Solids, J. Phys. Chem. Lett. 9, 4385 (2018).

[98] C. H. Hodges, Quantum Corrections to the Thomas-Fermi Approximation: The Kirzhnits Method, Can. J. Phys. 51, 1428 (1973).

[99] J. P. Perdew and L. A. Constantin, Laplacian-Level Density Functionals for the Kinetic Energy Density and Exchange-Correlation Energy, Phys. Rev. B 75, 155109 (2007).

[100] V. V. Karasiev, R. S. Jones, S. B. Trickey, and F. E. Harris, Properties of Constraint-Based Single-Point Approximate Kinetic Energy Functionals, Phys. Rev. B 80, 245120 (2009).

[101] S. Laricchia, L. A. Constantin, E. Fabiano, and F. Della Sala, Laplacian-Level Kinetic Energy Approximations Based on the Fourth-Order Gradient Expansion: Global Assessment and Application to the Subsystem Formulation of Density Functional Theory, J. Chem. Theory Comput. 10, 164 (2014).

[102] A. C. Cancio, D. Stewart, and A. Kuna, Visualization and Analysis of the Kohn-Sham Kinetic Energy Density and Its
Orbital-Free Description in Molecules, J. Chem. Phys. 144, 084107 (2016).

[103] J. Seino, R. Kageyama, M. Fujinami, Y. Ikabata, and H. Nakai, Semi-Local Machine-Learned Kinetic Energy Density Functional with Third-Order Gradients of Electron Density, J. Chem. Phys. 148, 241705 (2018).

[104] P. Golub and S. Manzhos, Kinetic Energy Densities Based on the Fourth Order Gradient Expansion: Performance in Different Classes of Materials and Improvement via Machine Learning, Phys. Chem. Chem. Phys. 21, 378 (2019).

[105] L. A. Constantin, E. Fabiano, and F. Della Sala, Performance of Semilocal Kinetic Energy Functionals for Orbital-Free Density Functional Theory, J. Chem. Theory Comput. 15, 3044 (2019).

[106] P. J. Feibelman, Surface Electromagnetic Fields, Prog. Surf. Sci. 12, 287 (1982).

[107] M. J. Stott and E. Zaremba, Linear-Response Theory within the Density-Functional Formalism: Application to Atomic Polarizabilities, Phys. Rev. A 21, 12 (1980).

[108] F. Della Sala, E. Fabiano, and L. A. Constantin, KineticEnergy-Density Dependent Semilocal Exchange-Correlation Functionals, Int. J. Quantum Chem. 116, 1641 (2016).

[109] D. A. Kirzhnits, Quantum Corrections to the ThomasFermi Equation, Sov. Phys. JETP 5, 64 (1957), http://www .jetp.ac.ru/cgi-bin/dn/e_005_01_0064.pdf.

[110] See Supplemental Material at http://link.aps.org/ supplemental/10.1103/PhysRevX.11.011049 for a schematic image of simulation domain for a nanosphere and nanosphere dimer; derivation of Eq. (10) for the functional derivative $\delta T_{s} / \delta n$ of noninteracting KE functional $T_{s}$ and Eq. (12) for the first-order term of the potential $\left(\delta T_{s} / \delta n\right)_{1}$; derivation of Eq. (19) for the asymptotic form of the Laplacian term for spherical systems; details of the fitting procedure to extract the oscillator strength from the absorption spectra of $\mathrm{Na}$ jellium nanospheres; additional figures and tables concerning results for the PGSL and PGSLN functionals.

[111] J. P. Perdew and A. Zunger, Self-Interaction Correction to Density-Functional Approximations for Many-Electron Systems, Phys. Rev. B 23, 5048 (1981).

[112] COMSOL Multiphysics, https://www.comsol.com.

[113] C. Ciracì, Y. A. Urzhumov, and D. R. Smith, Effects of Classical Nonlocality on the Optical Response of ThreeDimensional Plasmonic Nanodimers, J. Opt. Soc. Am. B 30, 2731 (2013).

[114] C. Ciracì, Y. Urzhumov, and D. R. Smith, Far-Field Analysis of Axially Symmetric Three-Dimensional Directional Cloaks, Opt. Express 21, 9397 (2013).

[115] O. Bohigas, A. Lane, and J. Martorell, Sum Rules for Nuclear Collective Excitations, Phys. Rep. 51, 267 (1979).

[116] Z.-J. Yang, T. J. Antosiewicz, R. Verre, F. J. G. de Abajo, S. P. Apell, and M. Käll, Ultimate Limit of Light Extinction by Nanophotonic Structures, Nano Lett. 15, 7633 (2015).

[117] S. Smiga, E. Fabiano, L. A. Constantin, and F. Della Sala, Laplacian-Dependent Models of the Kinetic Energy Density: Applications in Subsystem Density Functional Theory with Meta-Generalized Gradient Approximation Functionals, J. Chem. Phys. 146, 064105 (2017).

[118] L. A. Constantin, E. Fabiano, and F. Della Sala, Modified Fourth-Order Kinetic Energy Gradient Expansion with 
Hartree Potential-Dependent Coefficients, J. Chem. Theory Comput. 13, 4228 (2017).

[119] J. A. Scholl, A. L. Koh, and J.A. Dionne, Quantum Plasmon Resonances of Individual Metallic Nanoparticles, Nature (London) 483, 421 (2012).

[120] K. Ding and C. T. Chan, An Eigenvalue Approach to Quantum Plasmonics Based on a Self-Consistent Hydrodynamics Method, J. Phys. Condens. Matter 30, 084007 (2018).

[121] O. Pérez-González, N. Zabala, A. G. Borisov, N. J. Halas, P. Nordlander, and J. Aizpurua, Optical Spectroscopy of Conductive Junctions in Plasmonic Cavities, Nano Lett. 10, 3090 (2010).

[122] H.-H. Jeong, M. C. Adams, J.-P. Günther, M. AlarcónCorrea, I. Kim, E. Choi, C. Miksch, A. F. Mark, A. G. Mark, and P. Fischer, Arrays of Plasmonic Nanoparticle Dimers with Defined Nanogap Spacers, ACS Nano 13, 11453 (2019).
[123] L. Schumacher, J. Jose, D. Janoschka, P. Dreher, T. J. Davis, M. Ligges, R. Li, M. Mo, S. Park, X. Shen, S. Weathersby, J. Yang, X. Wang, F. Meyer zu Heringdorf, K. Sokolowski-Tinten, and S. Schlücker, Precision Plasmonics with Monomers and Dimers of Spherical Gold Nanoparticles: Nonequilibrium Dynamics at the Time and Space Limits, J. Phys. Chem. C 123, 13181 (2019).

[124] A. Zangwill and P. Soven, Density-Functional Approach to Local-Field Effects in Finite Systems: Photoabsorption in the Rare Gases, Phys. Rev. A 21, 1561 (1980).

[125] W. Ekardt, Size-Dependent Photoabsorption and Photoemission of Small Metal Particles, Phys. Rev. B 31, 6360 (1985).

[126] G. Bertsch, An RPA Program for Jellium Spheres, Comput. Phys. Commun. 60, 247 (1990).

[127] E. Prodan and P. Nordlander, Electronic Structure and Polarizability of Metallic Nanoshells, Chem. Phys. Lett. 352, 140 (2002). 